\title{
Simulations of merging and squeezing bunches in booster and AGS
}

\author{
C.J. Gardner
}

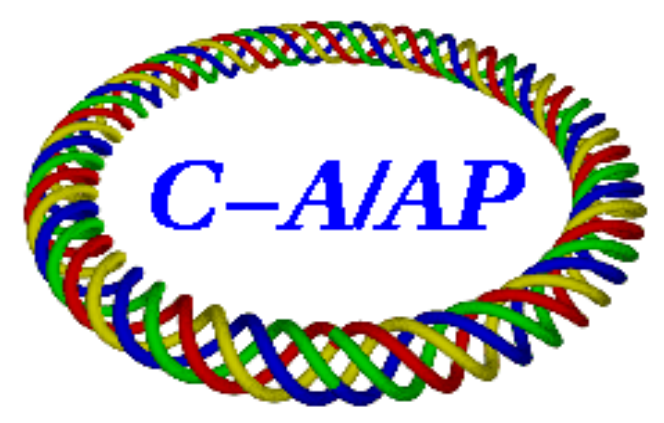

\section{Collider-Accelerator Department Brookhaven National Laboratory Upton, NY 11973}

Notice: This document has been authorized by employees of Brookhaven Science Associates, LLC under Contract No. DE-AC02-98CH10886 with the U.S. Department of Energy. The United States Government retains a nonexclusive, paid-up, irrevocable, world-wide license to publish or reproduce the published form of this document, or allow others to do so, for United States Government purposes. 


\title{
Simulations of Merging and Squeezing Bunches in Booster and AGS
}

\author{
C.J. Gardner
}

July 30, 2012

During the 2012 RHIC run, uranium, gold, and copper ions from EBIS were accelerated in Booster and AGS and delivered to RHIC for the physics program. In order to achieve the desired number of ions per bunch in RHIC, it was necessary to merge bunches in both Booster and AGS. After the bunches are merged in AGS, they need to be squeezed into harmonic 12 buckets for subsequent acceleration. In this note we carry out simulations of bunch merging and squeezing in Booster and AGS. These are meant to serve as benchmarks for what can be achieved in practice. In Booster the total longitudinal emittance of the 4 bunches to be merged is taken to be $0.15 \mathrm{eV}$ s per nucleon. In AGS the total for each group of 4 bunches to be merged is taken to be $0.60 \mathrm{eV}$ s per nucleon. The simulations show that the RF voltages currently available in Booster and AGS are sufficient for merging and holding the merged bunches in RF buckets. The voltages available in AGS are not sufficient for completely squeezing the merged bunches into harmonic 12 buckets.

The merging of bunches is treated in sections 9 through 12 . The required squeezing into harmonic 12 buckets is treated in sections 13 and 14 .

Debunching and rebunching beam as an alternative to merging bunches in Booster is treated in section 15. For completeness, sections 1 through 8 give a derivation of the turn-by-turn equations of longitudinal motion at constant magnetic field. The derivation is based on the work of MacLachlan [1]. The reader may wish to skip over these sections and start with section 9 . 


\section{Synchronous Parameters}

Let $2 \pi R$ and $\rho$ be the circumference and radius-of-curvature of the design orbit in a given ring, and let $B$ and $2 \pi R_{s}$ be the magnetic field and orbit circumference for the synchronous particle. We assume that $B$ and $R_{s}$ are given and calculate the other synchronous particle parameters in terms of these. The radius-of-curvature of the synchronous particle is

$$
\rho_{s}=\rho\left(R_{s} / R\right)^{1 / \alpha}
$$

where

$$
\alpha=\frac{1}{\gamma_{t}^{2}}
$$

is the "momentum compaction" factor and $\gamma_{t}$ is the transition gamma of the ring. The momentum of the synchronous particle is given by

$$
c p_{s}=e Q B \rho_{s}
$$

where $e$ is the proton charge and $e Q$ is the charge of the particle. The energy is

$$
E_{s}=\sqrt{\left(c p_{s}\right)^{2}+m^{2} c^{4}}
$$

where $m$ is the mass of the particle. The synchronous $\beta, \gamma$ and angular velocity are

$$
\beta_{s}=c p_{s} / E_{s}, \quad \gamma_{s}=E_{s} /\left(m c^{2}\right), \quad \omega_{s}=c \beta_{s} / R_{s} .
$$

The revolution period and frequency of the synchronous particle are

$$
T_{s}=\frac{2 \pi}{\omega_{s}}, \quad f_{s}=\frac{1}{T_{s}} .
$$

We also define the phase slip factor

$$
\eta_{s}=\frac{1}{\gamma_{t}^{2}}-\frac{1}{\gamma_{s}^{2}}
$$




\section{Time Equation}

We consider a ring with a single RF gap. Let $T_{n}^{s}$ and $T_{n}$ be respectively the times at which the synchronous and asynchronous particles make their $n$th pass through the gap. The synchronous particle experiences no acceleration in the gap and revolves around the ring with constant angular velocity $\omega_{s}$. Thus

$$
T_{n+1}^{s}=T_{n}^{s}+\frac{2 \pi}{\omega_{s}}
$$

and taking $T_{1}^{s}=0$ it follows that

$$
T_{n+1}^{s}=n T_{s} .
$$

Similarly, for the asynchronous particle we have

$$
T_{n+1}=T_{n}+\frac{2 \pi}{\omega_{n}}
$$

where $\omega_{n}$ is the angular velocity just after the $n$th pass through the gap.

Defining

$$
t_{n+1}=T_{n+1}-T_{n+1}^{s}, \quad t_{n}=T_{n}-T_{n}^{s}
$$

we then have

$$
t_{n+1}=t_{n}+2 \pi\left(\frac{1}{\omega_{n}}-\frac{1}{\omega_{s}}\right)=t_{n}+\left(\frac{\omega_{s}-\omega_{n}}{\omega_{n}}\right) T_{s} .
$$

\section{$3 \quad$ Energy Equation}

Now let $E_{n}$ be the energy of the asynchronous particle just after its $n$th pass through the gap. Then we have

$$
E_{n+1}=E_{n}+e Q V\left(T_{n+1}\right),
$$

where $V(T)$ is the voltage across the gap at time $T$. Since the synchronous particle undergoes no acceleration we must have

$$
V\left(T_{n}^{s}\right)=0
$$

for all $n$. We assume that

$$
V\left(T+T_{s} / h\right)=V(T)
$$


where $h$ is a positive integer called the fundamental harmonic. It then follows that

$$
V\left(T+n T_{s}\right)=V(T) .
$$

In terms of $E_{n}$, the other asynchronous parameters are

$$
c p_{n}=\sqrt{E_{n}^{2}-m^{2} c^{4}}, \quad \beta_{n}=c p_{n} / E_{n}
$$

and

$$
\rho_{n}=\frac{c p_{n}}{e Q B}, \quad R_{n}=R\left(\rho_{n} / \rho\right)^{\alpha}, \quad \omega_{n}=c \beta_{n} / R_{n} .
$$

Define

$$
e_{n+1}=E_{n+1}-E_{s}, \quad e_{n}=E_{n}-E_{s} .
$$

Then using

$$
T_{n+1}=t_{n+1}+T_{n+1}^{s}=t_{n+1}+n T_{s}
$$

we have

$$
V\left(T_{n+1}\right)=V\left(t_{n+1}\right)
$$

and

$$
e_{n+1}=e_{n}+e Q V\left(t_{n+1}\right) \text {. }
$$

This, together with

$$
t_{n+1}=t_{n}+\left(\frac{\omega_{s}-\omega_{n}}{\omega_{n}}\right) T_{s}
$$

gives the turn-by-turn longitudinal motion of the asynchronous particle.

\section{Symplectic Map}

The Jacobian matrix elements for the map from $\left(t_{n}, e_{n}\right)$ to $\left(t_{n+1}, e_{n+1}\right)$ are

$$
\begin{gathered}
\frac{\partial t_{n+1}}{\partial t_{n}}=1, \quad \frac{\partial t_{n+1}}{\partial e_{n}}=2 \pi \frac{\partial\left(1 / \omega_{n}\right)}{\partial e_{n}} \\
\frac{\partial e_{n+1}}{\partial t_{n}}=e Q V^{\prime}, \quad \frac{\partial e_{n+1}}{\partial e_{n}}=1+2 \pi e Q V^{\prime} \frac{\partial\left(1 / \omega_{n}\right)}{\partial e_{n}}
\end{gathered}
$$

where $V^{\prime}$ is the derivative of $V$ with respect to $T$ at time $T_{n+1}$. Thus we have

$$
\left(\frac{\partial t_{n+1}}{\partial t_{n}}\right)\left(\frac{\partial e_{n+1}}{\partial e_{n}}\right)-\left(\frac{\partial t_{n+1}}{\partial e_{n}}\right)\left(\frac{\partial e_{n+1}}{\partial t_{n}}\right)=1
$$

and the map is symplectic. This guarantees that the map preserves area. 


\section{Approximate Time Equation}

Writing

$$
\frac{\omega_{s}}{\omega_{n}}=\left\{1+\left(\frac{\omega_{n}-\omega_{s}}{\omega_{s}}\right)\right\}^{-1}
$$

and expanding we have

$$
\frac{\omega_{s}}{\omega_{n}}=1-\left(\frac{\omega_{n}-\omega_{s}}{\omega_{s}}\right)+\left(\frac{\omega_{n}-\omega_{s}}{\omega_{s}}\right)^{2}-\left(\frac{\omega_{n}-\omega_{s}}{\omega_{s}}\right)^{3}+\cdots .
$$

To first order in $p_{n}-p_{s}$ and $E_{n}-E_{s}$ we also have

$$
\left(\frac{\omega_{n}-\omega_{s}}{\omega_{s}}\right)=-\eta_{s}\left(\frac{p_{n}-p_{s}}{p_{s}}\right)=-\eta_{s}\left(\frac{E_{n}-E_{s}}{\beta_{s}^{2} E_{s}}\right) .
$$

Thus to first order

$$
\frac{\omega_{s}}{\omega_{n}}=1-\left(\frac{\omega_{n}-\omega_{s}}{\omega_{s}}\right)=1+\left(\frac{\eta_{s}}{\beta_{s}^{2} E_{s}}\right) e_{n}
$$

and (23) becomes

$$
t_{n+1}=t_{n}+T_{s}\left(\frac{\eta_{s}}{\beta_{s}^{2} E_{s}}\right) e_{n}
$$

This together with

$$
e_{n+1}=e_{n}+e Q V\left(t_{n+1}\right)
$$

again produces a symplectic map from $\left(t_{n}, e_{n}\right)$ to $\left(t_{n+1}, e_{n+1}\right)$.

\section{$6 \quad$ Phase and $W$ Equations}

Let us now introduce new variables

$$
\phi_{n}=h \omega_{s} t_{n}, \quad W_{n}=\frac{e_{n}}{h \omega_{s}} .
$$

Here $\phi_{n}$ is the phase that corresponds to time $t_{n}$, and $W_{n}$ is defined so that the transformation from $\left(t_{n}, e_{n}\right)$ to $\left(\phi_{n}, W_{n}\right)$ is symplectic. Note that $\phi_{n}$ varies from $-\pi$ to $+\pi$ as $t_{n}$ varies from $-T_{s} /(2 h)$ to $+T_{s} /(2 h)$. In terms of the new variables equations (31) and (32) become

$$
\phi_{n+1}=\phi_{n}+T_{s}\left(\frac{h^{2} \omega_{s}^{2} \eta_{s}}{\beta_{s}^{2} E_{s}}\right) W_{n}
$$


and

$$
W_{n+1}=W_{n}+T_{s}\left(\frac{e Q}{2 \pi h}\right) V\left(\frac{\phi_{n+1}}{h \omega_{s}}\right) .
$$

Defining

$$
a=\left(\frac{h^{2} \omega_{s}^{2} \eta_{s}}{\beta_{s}^{2} E_{s}}\right)
$$

and

$$
F\left(\phi_{n+1}\right)=\left(\frac{e Q}{2 \pi h}\right) V\left(\frac{\phi_{n+1}}{h \omega_{s}}\right)
$$

we then have

$$
\phi_{n+1}=\phi_{n}+a T_{s} W_{n}
$$

and

$$
W_{n+1}=W_{n}+T_{s} F\left(\phi_{n+1}\right) .
$$

These equations again generate a sympletic map. Note that since $V(0)=0$ and $V\left(T+T_{s} / h\right)=V\left(T_{s}\right)$ we have

$$
F(0)=0, \quad F(\phi+2 \pi)=F(\phi) .
$$

Since we are interested in the momentum deviation $p_{n}-p_{s}$, it is useful to have an expression for this in terms of $W_{n}$. Using (29) and (33) we find

$$
\left(\frac{p_{n}-p_{s}}{p_{s}}\right)=\left(\frac{E_{n}-E_{s}}{\beta_{s}^{2} E_{s}}\right)=\left(\frac{h \omega_{s}}{\beta_{s}^{2} E_{s}}\right) W_{n}
$$

\section{$7 \quad$ Fixed Points of the Motion}

Fixed points of the motion are points $\left(\phi_{f}, W_{f}\right)$ that are unchanged by (38) and (39). For these points we must have

$$
F\left(\phi_{f}\right)=0, \quad W_{f}=0 .
$$

Since $F(0)=0$ we see that the point $(0,0)$ is a fixed point.

If a particle launched near a fixed point stays close to the fixed point, the fixed point is said to be stable. In the Appendix we show that below transition $(a<0)$ a fixed point is stable if

$$
F^{\prime}\left(\phi_{f}\right)>0
$$

and unstable if

$$
F^{\prime}\left(\phi_{f}\right)<0
$$


where the primes denote differentiation with respect to $\phi$. Similarly above transition $(a>0)$ a fixed point will be stable if

$$
F^{\prime}\left(\phi_{f}\right)<0
$$

and unstable if

$$
F^{\prime}\left(\phi_{f}\right)>0
$$

\section{RF Buckets}

Consider the hamiltonian

$$
H(\phi, W)=\frac{1}{2} a W^{2}+U(\phi)
$$

where

$$
\frac{\partial U}{\partial \phi}=-F(\phi)
$$

The equations of motion are

$$
\begin{gathered}
\frac{d \phi}{d t}=\frac{\partial H}{\partial W}=a W \\
\frac{d W}{d t}=-\frac{\partial H}{\partial \phi}=F(\phi)
\end{gathered}
$$

and we have

$$
\frac{d H}{d t}=\frac{\partial H}{\partial t}=\frac{\partial U}{\partial t} .
$$

The hamiltonian is therefore a constant of the motion if $U$ has no explicit dependence on time. This constraint can be used to determine the extent of the regions of bounded motion generated by $H$.

The fixed points are the same as those for the motion generated by (38) and (39). They are given by (42). Let

$$
U_{\phi \phi}=U^{\prime \prime}\left(\phi_{f}\right)
$$

where the primes denote differentiation with respect to $\phi$. Then below transition $(a<0)$ the fixed point will be stable if $U_{\phi \phi}<0$ and unstable if $U_{\phi \phi}>0$. Similarly above transition $(a>0)$ the fixed point will be stable if $U_{\phi \phi}>0$ and unstable if $U_{\phi \phi}<0$. 
Let $H_{u}$ be the value of $H$ at an unstable fixed point $\left(\phi_{u}, 0\right)$. Then

$$
H_{u}=U\left(\phi_{u}\right)
$$

and the equation

$$
H(\phi, W)=H_{u}
$$

can be solved to obtain

$$
W^{2}(\phi)=\frac{2}{a}\left\{U\left(\phi_{u}\right)-U(\phi)\right\} .
$$

The curve $W(\phi)$ is called the separatrix. Differentiating $W^{2}(\phi)$ with respect to $\phi$ we obtain

$$
\frac{d W^{2}}{d \phi}=-\frac{2}{a} U^{\prime}(\phi)
$$

and

$$
\frac{d^{2} W^{2}}{d^{2} \phi}=-\frac{2}{a} U^{\prime \prime}(\phi)
$$

which shows that $W^{2}(\phi)$ reaches a local maximum at each stable fixed point. The area around a stable fixed point and bounded by the separatrix is an RF bucket. This is the extent of the region of bounded motion around the fixed point. The beam contained in the RF bucket is called a bunch. The height $W_{b}$ of the bucket is given by

$$
W_{b}^{2}=\frac{2}{a}\left\{U\left(\phi_{u}\right)-U\left(\phi_{s}\right)\right\}
$$

where $\phi_{s}$ is the stable fixed point phase.

First-order symplectic integration [2] of (49) and (50) from time $t$ to time $t+T_{s}$ yields

$$
\phi\left(t+T_{s}\right)=\phi(t)+a T_{s} W(t)
$$

and

$$
W\left(t+T_{s}\right)=W(t)+T_{s} F\left(\phi\left(t+T_{s}\right)\right) .
$$

These are the same as (38) and (39). This suggests that the motion generated by the hamiltonian approximates that generated by (38) and (39). To the extent that this is true, the regions of bounded motion generated by $H$ will approximate those generated by (38) and (39). 


\section{Capturing Unbunched Beam into RF Buckets}

For simulations that start with bunched beam we want an initial beam distribution that consists of 4 bunches in 4 adjacent harmonic $4 h$ buckets. To obtain this we start with a uniform distribution of unbunched beam consisting of an 80-by- 80 array of particles which cover the region

$$
-\phi_{I} \leq \phi \leq \phi_{I}, \quad-W_{I} \leq W \leq W_{I}
$$

where

$$
\phi_{I}=\pi
$$

and

$$
W_{I}=\frac{E_{I}}{h \omega_{s}}=\frac{E_{I} T_{s}}{2 \pi h} .
$$

Here $E_{I}$ is the energy half-width of the unbunched beam. Note that according to (33) the phase $\phi$ varies from $-\pi$ to $+\pi$ as the time deviation $t$ varies from $-T_{s} /(2 h)$ to $+T_{s} /(2 h)$. This means that the region defined by (61) occupies $1 / h$ of the ring. The area of the region is

$$
\epsilon=\left(2 \phi_{I}\right)\left(2 W_{I}\right)=2 E_{I} T_{s} / h .
$$

This is the longitudinal emittance of the unbunched beam (in $1 / h$ of the ring).

To capture the beam into 4 adjacent harmonic $4 h$ buckets we take

$$
F(\phi)=-A \sin 4 \phi
$$

in (38) and (39). During capture $A$ is raised slowly and quadratically from zero to $A_{C}$ over time interval $0 \leq T \leq T_{C}$ according to

$$
A(T)=A_{C}\left(\frac{T}{T_{C}}\right)^{2}
$$

where

$$
A_{C}=\frac{e Q V_{C}}{2 \pi h}
$$

and $V_{C}$ is the $\mathrm{RF}$ harmonic $4 h$ voltage at time $T_{C}$. The time $T_{C}$ is chosen to be long enough to ensure that the longitudinal emittance of the 4 bunches is as close to that of the initial distribution of unbunched beam as practical. 


\section{Formulae for Merging 4 Bunches into 1}

To simulate the merge of 4 bunches into 1 we take

$$
F(\phi)=A_{1} \sin \phi-A_{2} \sin 2 \phi-A_{4} \sin 4 \phi
$$

in (38) and (39). The parameters $A_{1}, A_{2}$ and $A_{4}$ are either zero or positive. They are varied slowly during the merging process. Note again that the phase $\phi$ varies from $-\pi$ to $+\pi$ as the time deviation $t$ varies from $-T_{s} /(2 h)$ to $+T_{s} /(2 h)$.

In order to satisfy (48) we take

$$
U(\phi)=A_{1} \cos \phi-\frac{1}{2} A_{2} \cos 2 \phi-\frac{1}{4} A_{4} \cos 4 \phi
$$

which gives

$$
U^{\prime \prime}(\phi)=-A_{1} \cos \phi+2 A_{2} \cos 2 \phi+4 A_{4} \cos 4 \phi .
$$

This is positive at phases

$$
\phi_{u}= \pm \pi
$$

which shows that these are unstable fixed point phases below transition. Using (71) in (69) gives

$$
U\left(\phi_{u}\right)=-A_{1}-\frac{A_{2}}{2}-\frac{A_{4}}{4}
$$

which can be used in (55) to obtain the separatrix.

Starting with 4 bunches in 4 adjacent harmonic $4 h$ buckets, the merge is done in two steps. First the 4 bunches are merged into 2 and then the 2 are merged into 1 . The merge of 4 bunches into 2 takes place over the time interval $0 \leq T \leq T_{K}$. During this time $A_{1}$ is held at zero, $A_{2}$ is raised linearly from zero to $A_{K}$, and $A_{4}$ is lowered linearly from $A_{C}$ to zero. Thus

$$
A_{1}(T)=0
$$

and

$$
A_{2}(T)=A_{K}\left(\frac{T}{T_{K}}\right), \quad A_{4}(T)=A_{C}\left(\frac{T_{K}-T}{T_{K}}\right) .
$$

Here

$$
A_{K}=\frac{e Q V_{K}}{2 \pi h}, \quad A_{C}=\frac{e Q V_{C}}{2 \pi h}
$$


where $V_{K}$ is the harmonic $2 h$ voltage at time $T=T_{K}$ and $V_{C}$ is the harmonic $4 h$ voltage at time $T=0$.

The merge of 2 bunches into 1 takes place over the time interval $T_{K} \leq T \leq T_{L}$. During this time $A_{4}$ is held at zero, $A_{1}$ is raised linearly from zero to $A_{L}$, and $A_{2}$ is lowered linearly from $A_{K}$ to zero. Thus

$$
A_{1}(T)=A_{L}\left(\frac{T-T_{K}}{T_{L}-T_{K}}\right), \quad A_{2}(T)=A_{K}\left(\frac{T_{L}-T}{T_{L}-T_{K}}\right)
$$

and

$$
A_{4}(T)=0
$$

Here

$$
A_{L}=\frac{e Q V_{L}}{2 \pi h}
$$

where $V_{L}$ is the harmonic $h$ voltage at time $T_{L}$.

\section{Merging Bunches in Booster}

Beam from EBIS is injected into Booster and captured into 4 harmonic 4 buckets. The resulting 4 bunches are accelerated to a constant-field porch where they are merged into 1 bunch. Capture and acceleration to the porch are done with the A3 and B3 RF cavities. For Au32+ ions the harmonic 4 frequency on the porch is $4 f_{s}=1.860 \mathrm{MHz}$. The merge from 4 to 2 bunches is done with the A6 and E6 cavities operating at frequency $2 f_{s}=0.930 \mathrm{MHz}$. The merge from 2 to 1 bunches is then done with the A3 and B3 cavities operating at frequency $f_{s}=0.465 \mathrm{MHz}$.

To simulate the merges we start with 4 bunches of Au32+ ions on the porch. These are obtained from a uniform distribution of unbunched beam as described in section 9. Here we take fundamental harmonic $h=1$. We use capture time $T_{C}=30 \mathrm{~ms}$ and harmonic 4 voltage $V_{C}=2.0 \mathrm{kV}$ in (65), (66) and (67). The longitudinal emittance of the initial distribution of unbunched beam is taken to be $0.15 \mathrm{eV} \mathrm{s}$ per nucleon. Figure 1 shows the resulting bunches in the 4 buckets on the porch. One can see by inspection that the emittance of the 4 bunches is close to that of the initial distribution.

The merge from 4 to 2 bunches is shown in Figures 2 and 3. Here the harmonic 4,2 , and 1 voltages were programmed according to (73), (74) and (75) with the harmonic 4 voltage going from $V_{C}=2.0 \mathrm{kV}$ to zero, the 
harmonic 2 voltage going from zero to $V_{K}=1.0 \mathrm{kV}$, and the harmonic 1 voltage held at $V_{L}=0$. The merge time was $T_{K}=7.5 \mathrm{~ms}$.

The merge from 2 to 1 bunches is shown in Figures 4 and $\mathbf{5}$. Here the harmonic 4, 2, and 1 voltages were programmed according to (76), (77), and (78) with the harmonic 2 voltage going from $V_{K}=1.0 \mathrm{kV}$ to zero, the harmonic 1 voltage going from zero to $V_{L}=1.0 \mathrm{kV}$, and the harmonic 4 voltage held at $V_{C}=0$. The merge time was $T_{L}-T_{K}=7.5 \mathrm{~ms}$.

The final merged bunch shown in Figure 5 has tails which makes the effective emittance larger than that of the initial 4 bunches. This is a result of the short total merging time of $15 \mathrm{~ms}$. In practice we are limited to this time in order to avoid introducing potentially harmful harmonics on the local power grid. Figure 6 shows the final bunch for the case in which the two merge times are doubled. The effective emittance in this case is noticeably reduced.

\section{Merging Bunches on AGS Injection Porch}

Bunches from Booster are injected into harmonic 16 buckets on the AGS injection porch. Each group of 4 adjacent bunches is then merged into 2 bunches by bringing on harmonic 8 voltage with RF cavity KL while reducing the harmonic 16 voltage. The 2 bunches are then merged into 1 by bringing on harmonic 4 voltage with RF cavity L10 while reducing the harmonic 8 voltage. For Au77+ ions the harmonic 16 frequency on the porch is $16 f_{s}=2.61 \mathrm{MHz}$.

To simulate the merges we start with 4 adjacent bunches of Au77+ ions on the injection porch. These are again obtained from a uniform distribution of unbunched beam as described in section 9 . Here we take fundamental harmonic $h=4$. We use capture time $T_{C}=50 \mathrm{~ms}$ and harmonic 16 voltage $V_{C}=40 \mathrm{kV}$ in (65), (66) and (67). The longitudinal emittance of the unbunched beam is taken to be $0.60 \mathrm{eV}$ s per nucleon. Figure 7 shows the resulting bunches in 4 buckets on the porch. One can see by inspection that the emittance of the 4 bunches is close to that of the initial distribution.

The merge from 4 to 2 bunches is shown in Figures 8 and $\mathbf{9}$. Here the harmonic 16, 8, and 4 voltages were programmed according to (73), (74) and (75) with the harmonic 16 voltage going from $V_{C}=40 \mathrm{kV}$ to zero, the harmonic 8 voltage going from zero to $V_{K}=22 \mathrm{kV}$, and the harmonic 4 
voltage held at $V_{L}=0$. The merge time was $T_{K}=15 \mathrm{~ms}$.

The merge from 2 to 1 bunches is shown in Figures $\mathbf{1 0}$ and 11. Here the harmonic 16, 8, and 4 voltages were programmed according to (76), (77), and (78) with the harmonic 8 voltage going from $V_{K}=22 \mathrm{kV}$ to zero, the harmonic 4 voltage going from zero $V_{L}=22 \mathrm{kV}$, and the harmonic 16 voltage held at $V_{C}=0$. The merge time was $T_{L}-T_{K}=15 \mathrm{~ms}$. The total time for merging the 4 bunches into 1 bunch was $T_{L}=30 \mathrm{~ms}$.

One can see by inspection that the emittance of the final merged bunch shown in Figure 11 is close to that (0.60 eV s per nucleon) of the initial distribution of unbunched beam. It is easily held in the harmonic 4 bucket with voltage $V_{L}=22 \mathrm{kV}$. For subsequent acceleration this bunch needs to be squeezed into a harmonic 12 bucket. This is discussed in the next two sections.

\section{Formulae for Squeezing Bunches}

To simulate the squeezing of a harmonic $h$ bunch into a harmonic $3 h$ bucket we take

$$
F(\phi)=A_{1} \sin \phi+A_{2} \sin 2 \phi+A_{3} \sin 3 \phi
$$

in (38) and (39). The parameters $A_{1}, A_{2}$ and $A_{3}$ are either zero or positive. They are varied slowly during the squeezing process. Note again that the phase $\phi$ varies from $-\pi$ to $+\pi$ as the time deviation $t$ varies from $-T_{s} /(2 h)$ to $+T_{s} /(2 h)$.

In order to satisfy (48) we take

$$
U(\phi)=A_{1} \cos \phi+\frac{1}{2} A_{2} \cos 2 \phi+\frac{1}{3} A_{3} \cos 3 \phi
$$

which gives

$$
U^{\prime \prime}(\phi)=-A_{1} \cos \phi-2 A_{2} \cos 2 \phi-3 A_{3} \cos 3 \phi .
$$

This is positive at phases

$$
\phi_{u}= \pm \pi
$$

provided

$$
3 A_{3}>2 A_{2}-A_{1} \text {. }
$$


These are then unstable fixed point phases below transition. Evaluating (80) at phases (82) gives

$$
U\left(\phi_{u}\right)=-A_{1}+\frac{A_{2}}{2}-\frac{A_{3}}{3}
$$

which can be used in (55) to obtain the associated separatrix.

Now using

$$
\sin 2 \phi=2 \cos \phi \sin \phi
$$

and

$$
\sin 3 \phi=3 \sin \phi-4 \sin ^{3} \phi
$$

we have

$$
F(\phi)=\left\{A_{1}+2 A_{2} \cos \phi+A_{3}\left(4 \cos ^{2} \phi-1\right)\right\} \sin \phi .
$$

This shows that in addition to the unstable fixed point phases (82) we have fixed point phases given by

$$
A_{1}+2 A_{2} \cos \phi_{f}+A_{3}\left(4 \cos ^{2} \phi_{f}-1\right)=0 .
$$

For the case $A_{3}=0$ we have

$$
A_{1}+2 A_{2} \cos \phi_{f}=0
$$

which has a solution provided

$$
2 A_{2} \geq A_{1}
$$

Using

$$
\cos 2 \phi_{f}=2 \cos ^{2} \phi_{f}-1
$$

we then have

$$
U^{\prime \prime}\left(\phi_{f}\right)=\frac{1}{2 A_{2}}\left(2 A_{2}-A_{1}\right)\left(2 A_{2}+A_{1}\right)
$$

which is positive. The fixed point given by (89) is then unstable below transition. Evaluating (80) at this point gives

$$
U\left(\phi_{f}\right)=-\frac{1}{4 A_{2}}\left(A_{1}^{2}+2 A_{2}^{2}\right)
$$

which can be used in (55) to obtain the associated separatrix. 
For the case $A_{3} \neq 0$ the fixed point phases are given by

$$
\cos \phi_{f}=-\frac{A_{2}}{4 A_{3}} \pm \frac{1}{4 A_{3}} \sqrt{A_{2}^{2}+4 A_{3}\left(A_{3}-A_{1}\right)} .
$$

To evaluate $U(\phi)$ and $U^{\prime \prime}(\phi)$ at $\phi_{f}$ we use

$$
\cos 2 \phi=2 \cos ^{2} \phi-1
$$

and

$$
\cos 3 \phi=4 \cos ^{3} \phi-3 \cos \phi
$$

which give

$$
U(\phi)=A_{1} \cos \phi+\frac{A_{2}}{2}\left(2 \cos ^{2} \phi-1\right)+\frac{A_{3}}{3}\left(4 \cos ^{3} \phi-3 \cos \phi\right)
$$

and

$$
U^{\prime \prime}(\phi)=-A_{1} \cos \phi-2 A_{2}\left(2 \cos ^{2} \phi-1\right)-3 A_{3}\left(4 \cos ^{3} \phi-3 \cos \phi\right) .
$$

Using (88) we then have

$$
U^{\prime \prime}\left(\phi_{f}\right)=2 A_{2} \cos ^{2} \phi_{f}+2\left(3 A_{3}+A_{1}\right) \cos \phi_{f}+2 A_{2}
$$

which is positive if and only if

$$
\left\{2 A_{2} \cos \phi_{f}+\left(3 A_{3}+A_{1}\right)\right\}^{2}>\left(3 A_{3}+A_{1}\right)^{2}-4 A_{2}^{2} .
$$

For the values of $A_{1}, A_{2}$, and $A_{3}$ considered in this note, $U^{\prime \prime}\left(\phi_{f}\right)$ is positive for the phase given by the upper sign in (94). This is then the phase for an unstable fixed point below transition. The lower sign gives the phase for a stable fixed point below transition. Using

$$
\cos \phi_{u}=-\frac{A_{2}}{4 A_{3}}+\frac{1}{4 A_{3}} \sqrt{A_{2}^{2}+4 A_{3}\left(A_{3}-A_{1}\right)}
$$

in (97) gives $U\left(\phi_{u}\right)$ which can be used in (55) to obtain the associated separatrix.

The squeeze starts at time $T_{L}$ with

$$
A_{1}=A_{L}, \quad A_{2}=0, \quad A_{3}=0 .
$$

Here

$$
A_{L}=\frac{e Q V_{L}}{2 \pi h}
$$


where $V_{L}$ is the harmonic $h$ voltage at time $T_{L}$.

The first part of the squeeze takes place over time interval $T_{L} \leq T \leq T_{M}$. During this time $A_{1}$ is held at $A_{L}, A_{3}$ is held at zero, and $A_{2}$ is raised linearly from zero to $A_{K}$. Thus

$$
A_{1}=A_{L}, \quad A_{2}=A_{K}\left(\frac{T-T_{L}}{T_{M}-T_{L}}\right), \quad A_{3}=0
$$

where

$$
A_{K}=\frac{e Q V_{K}}{2 \pi h}
$$

and $V_{K}$ is the harmonic $2 h$ voltage at time $T=T_{M}$.

The second (and final) part of the squeeze takes place over time interval $T_{M} \leq T \leq T_{N}$. During this time $A_{1}$ is held fixed at $A_{L}, A_{2}$ is held fixed at $A_{K}$, and $A_{3}$ is raised linearly from zero to $A_{A}$. Thus

$$
A_{1}=A_{L}, \quad A_{2}=A_{K}, \quad A_{3}=A_{A}\left(\frac{T-T_{M}}{T_{N}-T_{M}}\right)
$$

where

$$
A_{A}=\frac{e Q V_{A}}{2 \pi h}
$$

and $V_{A}$ is the harmonic $3 h$ voltage at time $T=T_{N}$.

\section{Squeezing Bunches on AGS Injection Porch}

Starting with the merged bunch in Figure 11, the harmonic 8 voltage is raised form zero to $V_{K}=22$ over time interval $T_{L} \leq T \leq T_{M}$ according to (104) with $T_{M}-T_{L}=20 \mathrm{~ms}$. The harmonic 4 voltage is held at $V_{L}=22$ $\mathrm{kV}$. The resulting bunch is shown in Figure 12. Here the separatrix has been calculated using (80) and (93). Figure 13 shows the harmonic 4 and harmonic 8 buckets which act together to make the separatrix. Note that the harmonic 8 bucket acting by itself is not large enough to contain the bunch.

The left and right sides of the bunch in Figures 12 and 13 lie outside the region $-\pi / 3 \leq \phi \leq \pi / 3$. This means that when the harmonic 12 voltage is brought on some of the particles in the bunch will end up in the harmonic 12 buckets on each side of the central bucket. This is shown in Figures 14 through 19 where the harmonic 12 voltage is raised from zero to $V_{A}=180$ $\mathrm{kV}$ over time $T_{N}-T_{M}=10 \mathrm{~ms}$ according to (106) and (107). Here the 
brown separatrix has been calculated using (80) and (84). The black separatrix has been calculated using (97) and (101). The fraction of Au77+ ions in the two outer buckets in Figure 19 is $\underline{0.089}$.

Close inspection of the central bunch in Figure 19 and the green ellipse in Figure 20 shows that the bunch and ellipse are rotated slightly clockwise with respect to the vertical axis. This is a feature of the motion generated by (38) and (39) and is discussed in the Appendix. Figure 21 shows the harmonic 4, 8, and 12 buckets which act together to make the black and brown separatrices.

If the simulation that produced Figures 7 through 19 is carried out with the maximum harmonic 4 voltage reduced to $V_{L}=18 \mathrm{kV}$, then one obtains the squeezed bunches shown in Figures 22 and 23. Here the fraction of Au77+ ions in the outer buckets has increased to $\underline{0.138}$.

Carrying out the simulation with the maximum harmonic 4 voltage reduced further, to $V_{L}=14 \mathrm{kV}$, one obtains the squeezed bunches shown in Figures 24 through 30. In Figure 24 we see that the bucket is just large enough to contain the bunch. As the harmonic 12 voltage is brought on, Au77+ ions begin leaking out of the central bucket as in the previous cases, but now they end up populating two buckets (instead of just one) on each side of the central bucket. This is shown in Figures 25 through 30. Here the fraction of Au77+ ions outside the central bucket has increased to $\underline{0.180}$. The fraction in the outermost buckets is 0.075 .

These simulations show that having a sufficiently high harmonic 4 voltage $V_{L}$ is critical for keeping the fraction of beam particles that end up outside the central harmonic 12 bucket as low as possible. $V_{L}=22 \mathrm{kV}$ is about as high as the L10 cavity voltage can go in practice. If this and the harmonic 8 voltage $V_{K}$ could be raised to $28 \mathrm{kV}$ then the simulation shows that the fraction outside the central bucket would be reduced to just $\underline{0.0043}$ for a bunch of Au77+ ions with a longitudinal emittance of $0.60 \mathrm{eV} \mathrm{s}$ per nucleon.

During the 2012 RHIC run the measured fraction [3, 4] of Au77+ ions outside the central harmonic 12 bucket on the AGS injection porch ranged from 0.080 to 0.120. The measured emittance [5] of the four Au77+ bunches before merging on the porch was $0.56 \mathrm{eV}$ s per nucleon. The measured emittance [6] of the bunches after merging but before the squeeze was also $0.56 \mathrm{eV}$ s per nucleon, showing that the 4-to-2 and 2-to-1 merges conserve emittance. 


\section{Debunching and Rebunching Beam in Booster}

An alternative to merging bunches on the Booster merging porch is to debunch the 4 bunches and rebunch the beam into 1 bunch. To simulate the debunching we use

$$
F(\phi)=-A \sin 4 \phi
$$

in (38) and (39) with $A$ lowered quadratically from $A_{D}$ to zero over time interval $0 \leq T \leq T_{D}$ according to

$$
A(T)=A_{D}\left(\frac{T_{D}-T}{T_{D}}\right)^{2}
$$

where

$$
A_{D}=\frac{e Q V_{D}}{2 \pi}
$$

and $V_{D}$ is the harmonic 4 voltage at time $T=0$. Figures 31,32 and $\mathbf{3 3}$ show the debunching of the 4 bunches on the merging porch with $V_{D}=2$ $\mathrm{kV}$ and debunching time $T_{D}=7.5 \mathrm{~ms}$.

To simulate the rebunching of the debunched beam into 1 bunch we use

$$
F(\phi)=A \sin \phi
$$

with $A$ raised quadratically from zero to $A_{E}$ over time interval $T_{D} \leq T \leq T_{E}$ according to

$$
A(T)=A_{E}\left(\frac{T-T_{D}}{T_{E}-T_{D}}\right)^{2}
$$

where

$$
A_{E}=\frac{e Q V_{E}}{2 \pi}
$$

and $V_{E}$ is the RF harmonic 1 voltage at time $T_{E}$. Figures 34 and 35 show the rebunching of the beam on the merging porch with $V_{E}=1 \mathrm{kV}$ and rebunching time $T_{E}-T_{D}=7.5 \mathrm{~ms}$.

Comparing the bunch in Figure 35 with the one in Figure 5 we see that the tails in $\mathbf{3 5}$ are longer, making the effective emittance greater. 


\section{Appendix}

For motion near a fixed point $\left(\phi_{f}, 0\right)$ we have

$$
F(\phi)=-k\left(\phi-\phi_{f}\right)
$$

where

$$
k=-F^{\prime}\left(\phi_{f}\right) .
$$

Equations (38) and (39) then become

$$
\phi_{n+1}-\phi_{f}=\left(\phi_{n}-\phi_{f}\right)+a T_{s} W_{n}
$$

and

$$
W_{n+1}=W_{n}-k T_{s}\left(\phi_{n}-\phi_{f}\right)-a k T_{s}^{2} W_{n}
$$

Defining

$$
\Phi=\phi-\phi_{f}
$$

and

$$
\mathbf{X}_{n+1}=\left(\begin{array}{c}
\Phi_{n+1} \\
W_{n+1}
\end{array}\right), \quad \mathbf{X}_{n}=\left(\begin{array}{c}
\Phi_{n} \\
W_{n}
\end{array}\right)
$$

we then have

$$
\mathbf{X}_{n+1}=\mathbf{M X}_{n}
$$

where

$$
\mathbf{M}=\left(\begin{array}{cc}
1 & a T_{s} \\
-k T_{s} & 1-a k T_{s}^{2}
\end{array}\right) .
$$

The matrix $\mathbf{M}$ has unit determinant and its trace is

$$
\mathcal{T}=2-a k T_{s}^{2} .
$$

It can be expressed in Courant-Snyder [7] form

$$
\mathbf{M}=\left(\begin{array}{cc}
C+\mathcal{A} S & \mathcal{B} S \\
-\mathcal{G} S & C-\mathcal{A} S
\end{array}\right)
$$

where

$$
\begin{gathered}
C=1-\frac{1}{2} a k T_{s}^{2}, \quad S^{2}=1-C^{2} \\
\mathcal{B} S=a T_{s}, \quad 2 \mathcal{A} S=a k T_{s}^{2}, \quad \mathcal{G} S=k T_{s}
\end{gathered}
$$

and

$$
\mathcal{B G}-\mathcal{A}^{2}=1
$$


Using (123) in (120) one finds that

$$
G\left(\Phi_{n+1}, W_{n+1}\right)=G\left(\Phi_{n}, W_{n}\right)
$$

where

$$
G(\Phi, W)=\mathcal{G} \Phi^{2}+2 \mathcal{A} \Phi W+\mathcal{B} W^{2} .
$$

This shows that $G(\Phi, W)$ is a conserved quantity for the turn-by-turn motion generated by (116) and (117).

We assume that $k<0$ below transition and $k>0$ above transition. Then since $a<0$ below transition and $a>0$ above transition, we have

$$
a k>0 .
$$

We assume further that $|k|$ is small enough to ensure that

$$
|\mathcal{T}|<2
$$

It then follows that $|C|<1$ and $S^{2}>0$. The parameters $S, \mathcal{A}, \mathcal{B}$, and $\mathcal{G}$ are then all real numbers. The sign of $S$ is chosen so that $\mathcal{B}>0$. We then have

$$
G(\Phi, W)=\mathcal{E}
$$

where $\mathcal{E}$ is a real number. With the help of (126) we have

$$
\Phi^{2}+(\mathcal{A} \Phi+\mathcal{B} W)^{2}=\mathcal{E} \mathcal{B}
$$

and

$$
W^{2}+(\mathcal{G} \Phi+\mathcal{A} W)^{2}=\mathcal{E} \mathcal{G} .
$$

The maximum possible $\Phi$ is then

$$
\Phi_{M}=\sqrt{\mathcal{E B}}
$$

and the corresponding $W$ is

$$
W\left(\Phi_{M}\right)=-\frac{\mathcal{A}}{\mathcal{B}} \Phi_{M}
$$

Similarly the maximum possible $W$ is

$$
W_{M}=\sqrt{\mathcal{E G}}
$$

and the corresponding $\Phi$ is

$$
\Phi\left(W_{M}\right)=-\frac{\mathcal{A}}{\mathcal{G}} W_{M} .
$$


Here

$$
-\frac{\mathcal{A}}{\mathcal{B}}=-\frac{1}{2} k T_{s}, \quad-\frac{\mathcal{A}}{\mathcal{G}}=-\frac{1}{2} a T_{s} .
$$

This analysis shows that under the conditions we have assumed, a particle launched close to the fixed point will stay close to the fixed point. The fixed point is then said to be stable.

We note here that (131) defines an ellipse with area $\pi \mathcal{E}$ and

Courant-Snyder parameters $\mathcal{A}, \mathcal{B}$ and $\mathcal{G}$. Since we have assumed that $k<0$ below transition we also have $\mathcal{A}<0$. The ellipse is therefore rotated clockwise with respect to the vertical axis. This can be seen in Figure 20 where the maximum value of $W$ for the particles in the central bunch has been used in (136) to obtain the green ellipse.

We note also that defining

$$
\mathcal{H}(\Phi, W)=\frac{S}{2 T_{s}} G(\Phi, W)
$$

and using the identities

$$
\frac{S \mathcal{B}}{2 T_{s}}=\frac{a}{2}, \quad \frac{2 \mathcal{A} S}{2 T_{s}}=\frac{1}{2} a k T_{s}, \quad \frac{S \mathcal{G}}{2 T_{s}}=\frac{k}{2}
$$

we have

$$
\mathcal{H}(\Phi, W)=\frac{1}{2} a W^{2}+\frac{1}{2} k \Phi^{2}+\frac{1}{2} a k T_{s} \Phi W .
$$

This is a conserved quantity for the motion generated by (116) and (117). It shows that the hamiltonian

$$
H=\frac{1}{2} a W^{2}+\frac{1}{2} k \Phi^{2}
$$

is not conserved. In fact we have

$$
H(\Phi, W)=\mathcal{H}(\Phi, W)-\frac{1}{2} a k T_{s} \Phi W
$$

which we can write as

$$
H(\Phi, W)=\frac{S \mathcal{E}}{2 T_{s}}-\frac{1}{2} a k T_{s} \Phi W
$$

where

$$
\mathcal{E}=G(\Phi, W) .
$$

The first term on the right hand side of (144) is constant but the second term varies with $\Phi$ and $W$. 
Now let

$$
\psi=\arcsin S
$$

where $S$ is given by (124). Then

$$
C=\cos \psi, \quad S=\sin \psi
$$

and following Courant and Snyder [7] we can write

$$
\mathbf{M}=\mathbf{I} \cos \psi+\mathbf{J} \sin \psi
$$

where

$$
\mathbf{I}=\left(\begin{array}{ll}
1 & 0 \\
0 & 1
\end{array}\right), \quad \mathbf{J}=\left(\begin{array}{rr}
\mathcal{A} & \mathcal{B} \\
-\mathcal{G} & -\mathcal{A}
\end{array}\right)
$$

Here

$$
\mathbf{J}^{2}=-\mathbf{I}
$$

and it follows that

$$
\{\mathbf{I} \cos \psi+\mathbf{J} \sin \psi\}\{\mathbf{I} \cos \chi+\mathbf{J} \sin \chi\}=\mathbf{I} \cos (\psi+\chi)+\mathbf{J} \sin (\psi+\chi) .
$$

Thus we have

$$
\mathbf{M}^{n}=\mathbf{I} \cos n \psi+\mathbf{J} \sin n \psi
$$

and

$$
\begin{gathered}
\Phi_{n}=\left(C_{n}+\mathcal{A} S_{n}\right) \Phi_{0}+\mathcal{B} S_{n} W_{0} \\
W_{n}=-\mathcal{G} S_{n} \Phi_{0}+\left(C_{n}-\mathcal{A} S_{n}\right) W_{0}
\end{gathered}
$$

where

$$
C_{n}=\cos n \psi, \quad S_{n}=\sin n \psi
$$

and $\Phi_{0}$ and $W_{0}$ are initial values of $\phi$ and $W$. Collecting terms we have

$$
\begin{gathered}
\Phi_{n}=\Phi_{0} C_{n}+\left(\mathcal{A} \Phi_{0}+\mathcal{B} W_{0}\right) S_{n} \\
W_{n}=W_{0} C_{n}-\left(\mathcal{G} \Phi_{0}+\mathcal{A} W_{0}\right) S_{n}
\end{gathered}
$$

and therefore

$$
\begin{aligned}
\Phi_{n} & =\sqrt{\mathcal{E B}}\left\{C_{n} \cos \xi-S_{n} \sin \xi\right\}=\sqrt{\mathcal{E B}} \cos (n \psi+\xi) \\
W_{n} & =\sqrt{\mathcal{E} \mathcal{G}}\left\{C_{n} \sin \chi+S_{n} \cos \chi\right\}=\sqrt{\mathcal{E G}} \sin (n \psi+\chi)
\end{aligned}
$$

where

$$
\mathcal{E B}=\Phi_{0}^{2}+\left(\mathcal{A} \Phi_{0}+\mathcal{B} W_{0}\right)^{2}
$$




$$
\mathcal{E} \mathcal{G}=W_{0}^{2}+\left(\mathcal{G} \Phi_{0}+\mathcal{A} W_{0}\right)^{2}
$$

and

$$
\begin{gathered}
\sqrt{\mathcal{E B}} \cos \xi=\Phi_{0}, \quad \sqrt{\mathcal{E B}} \sin \xi=-\left(\mathcal{A} \Phi_{0}+\mathcal{B} W_{0}\right) \\
\sqrt{\mathcal{E G}} \sin \chi=W_{0}, \quad \sqrt{\mathcal{E} \mathcal{G}} \cos \chi=-\left(\mathcal{G} \Phi_{0}+\mathcal{A} W_{0}\right) .
\end{gathered}
$$

These equations give explicitly the turn-by-turn motion near the stable fixed point.

Note that the synchrotron tune is defined to be

$$
Q_{s}=\frac{|\psi|}{2 \pi} \text {. }
$$

The synchrotron oscillation period is then

$$
P_{s}=\frac{T_{s}}{Q_{s}}
$$

For sufficiently small $a k T_{s}^{2}$ we have

$$
\psi=-T_{s} \sqrt{a k}
$$

which gives

$$
Q_{s}=\frac{T_{s}}{2 \pi} \sqrt{a k}, \quad P_{s}=\frac{2 \pi}{\sqrt{a k}}
$$

For the case

$$
F(\phi)=\left(\frac{e Q V_{G}}{2 \pi h}\right) \sin \phi
$$

we have stable fixed point phase $\phi_{s}=0$ (below transition) and

$$
a k=-\frac{e Q V_{G}}{2 \pi h}\left(\frac{h^{2} \omega_{s}^{2} \eta_{s}}{\beta_{s}^{2} E_{s}}\right)=-\frac{e Q V_{G}}{2 \pi h}\left(\frac{h^{2} c^{2} \eta_{s}}{R_{s}^{2} E_{s}}\right)
$$

For all of the above work we have assumed that $k<0$ below transition and $k>0$ above transition. If instead we assume that $k>0$ below transition and $k<0$ above transition then

$$
a k<0
$$

and we see from (122) that the trace of $\mathbf{M}$ is greater than 2. One then finds that a particle launched close to the fixed point will not stay close to the fixed point. The fixed point is then said to be unstable. 


\section{References}

[1] J.A. MacLachlan, "Difference Equations for Longitudinal Motion in a Synchrotron", Fermilab internal report FNAL FN-529, December 15, 1989; "Differential Equations for Longitudinal Motion in a Synchrotron", Fermilab internal report FNAL FN-532, January 25, 1990.

[2] H. Yoshida, Numerical Integration Methods, Handbook of Accelerator Physics and Engineering, Edited by A.W. Chao and M. Tigner, World Scientific, 1999, pp. 85-87.

[3] K.L. Zeno, Booster-AGS-EBIS-2012 elog entry 16:11, 29 May 2012.

[4] K.L. Zeno, Booster-AGS-EBIS-2012 elog entry 14:47, 19 June 2012.

[5] K.L. Zeno, Booster-AGS-EBIS-2012 elog entry 15:11, 19 June 2012.

[6] K.L. Zeno, Booster-AGS-EBIS-2012 elog entry 15:30, 19 June 2012.

[7] E.D. Courant and H.S. Snyder, Annals of Physics 3, 1-48 (1958). 


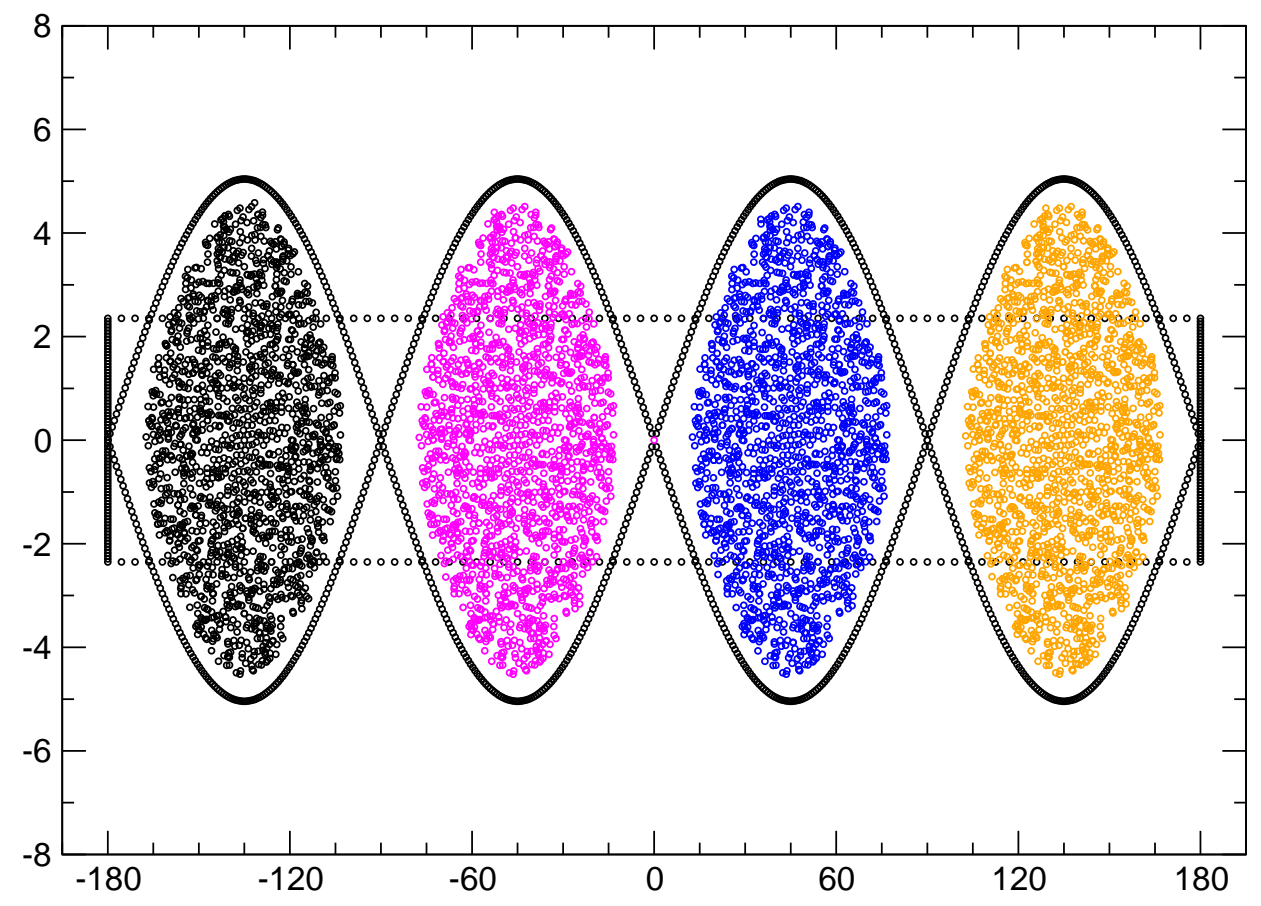

Figure 1: Four bunches of Au32+ ions in harmonic 4 buckets on the Booster merging porch. The harmonic 4 frequency is $4 f_{s}=1.860 \mathrm{MHz}$. The voltage is $V_{C}=2.0 \mathrm{kV}$. The black rectangle is the border of the uniform distribution used to make the bunches. The longitudinal emittance of the distribution is $0.15 \mathrm{eV}$ s per nucleon. One can see by inspection that the emittance of the 4 bunches is close to that of the initial distribution. The horizontal axis is the phase $\phi$ in degrees. The vertical axis is $W$ in units of eVs. 


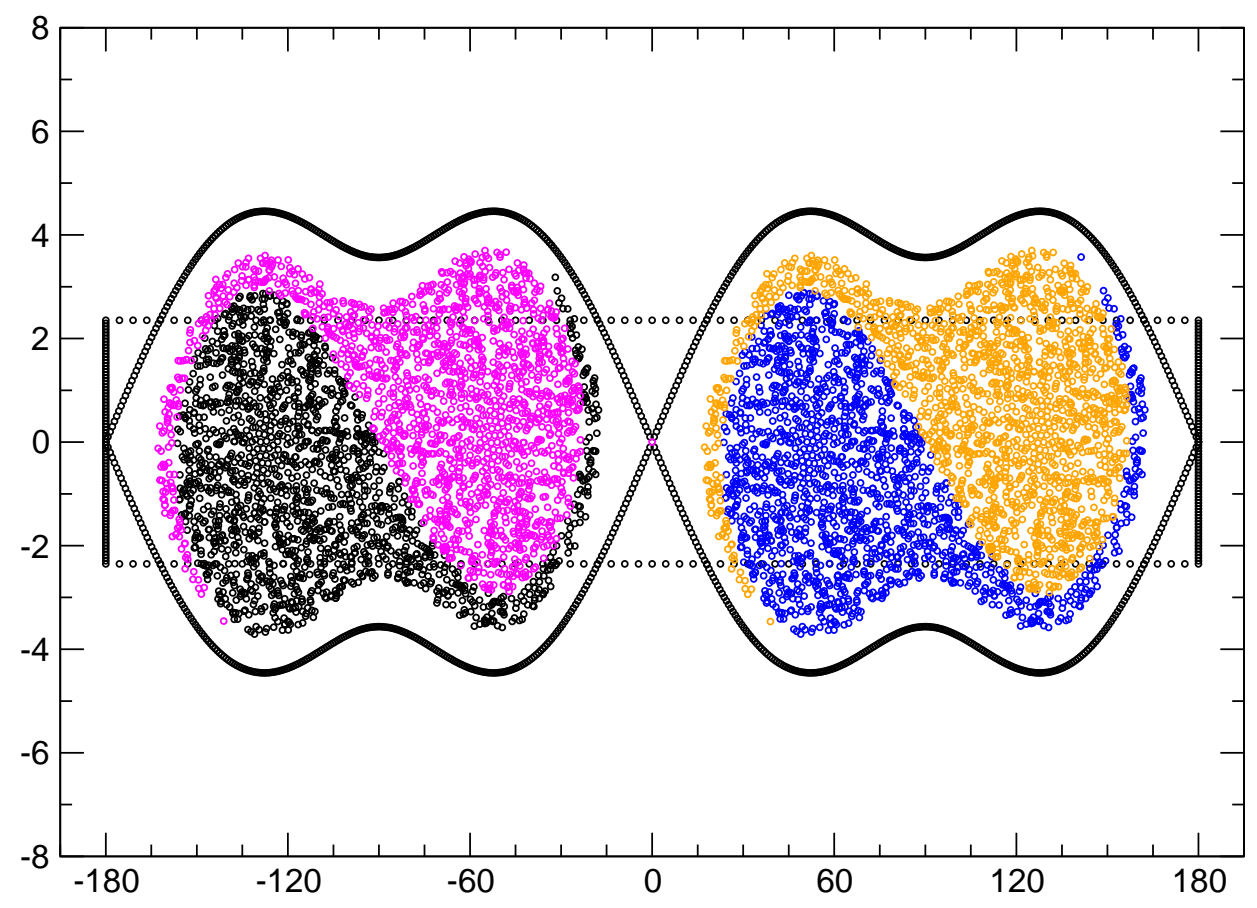

Figure 2: Halfway through the 4-to-2 merge of the bunches in Figure 1. Here the harmonic 4 voltage is $V_{C} / 2=1.0 \mathrm{kV}$. The harmonic 2 voltage is $V_{K} / 2=0.5 \mathrm{kV}$. The time from the start of the merge is $T_{K} / 2=7.5 / 2 \mathrm{~ms}$. 


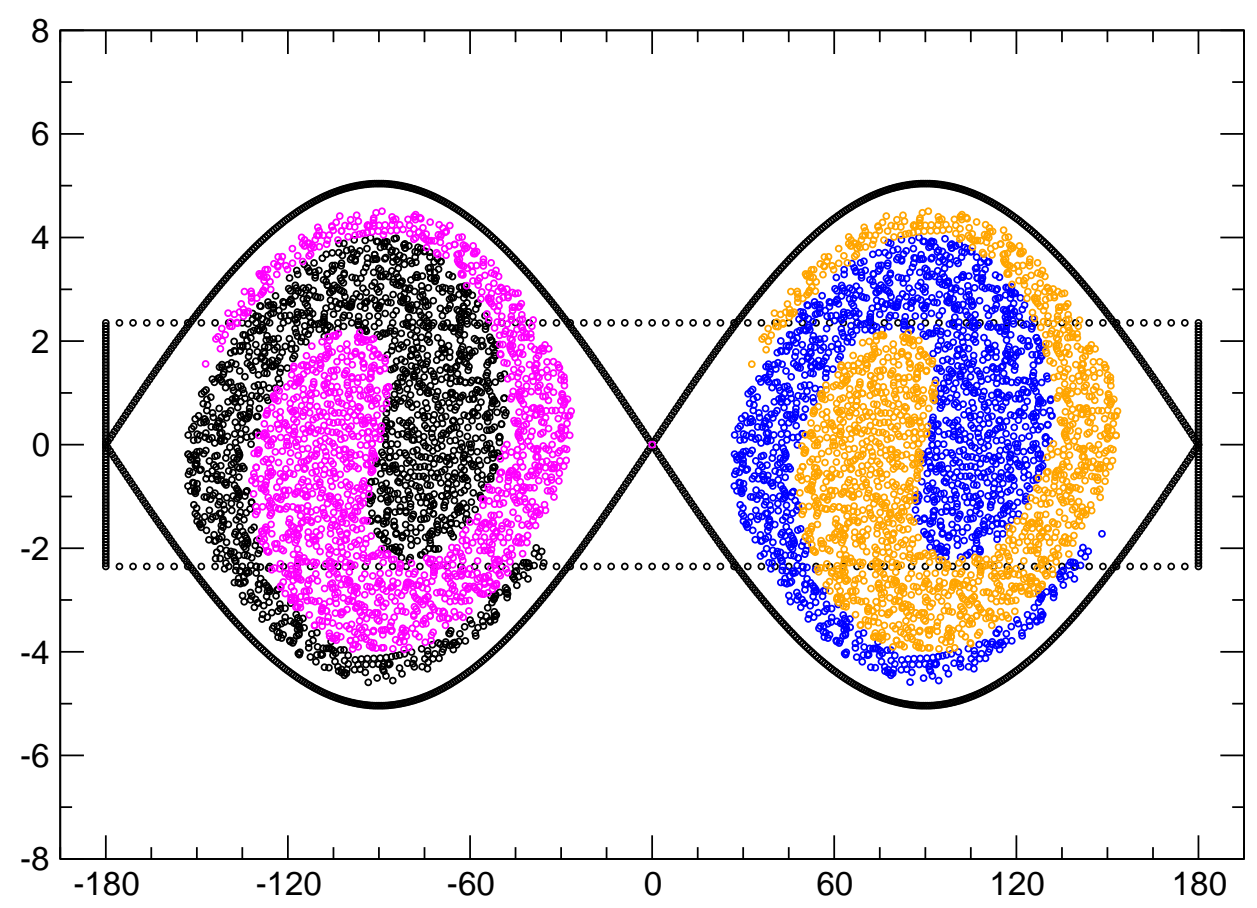

Figure 3: Completion of the 4-to-2 merge. Here the harmonic 2 voltage is $V_{K}=1.0 \mathrm{kV}$. The total time for the merge is $T_{K}=7.5 \mathrm{~ms}$. 


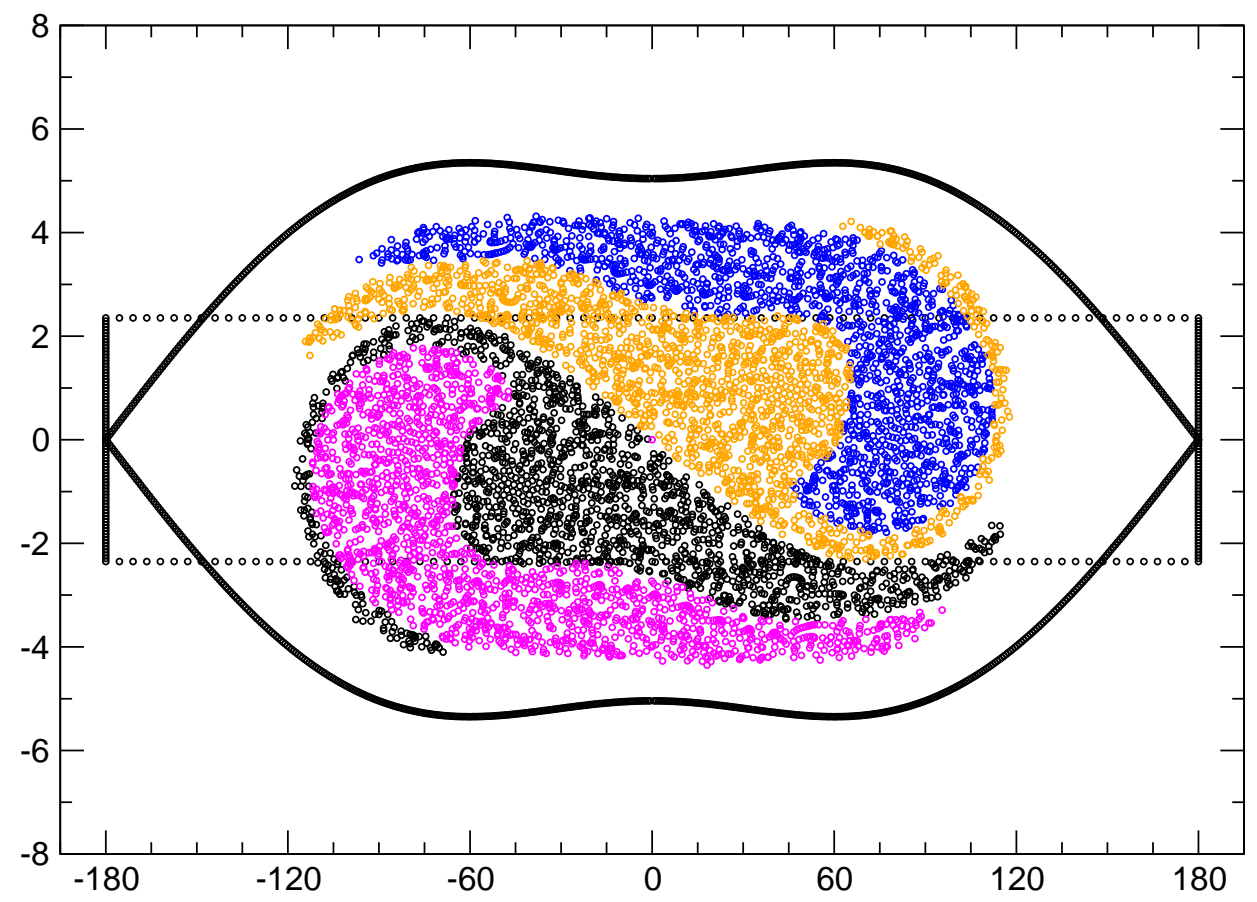

Figure 4: Halfway through the 2-to-1 merge of the bunches in Figure 3. Here the harmonic 2 voltage is $V_{K} / 2=0.5 \mathrm{kV}$. The harmonic 1 voltage is $V_{L} / 2=0.5 \mathrm{kV}$. The time from the start of the 2-to- 1 merge is $\left(T_{L}-T_{K}\right) / 2=$ $7.5 / 2 \mathrm{~ms}$. 


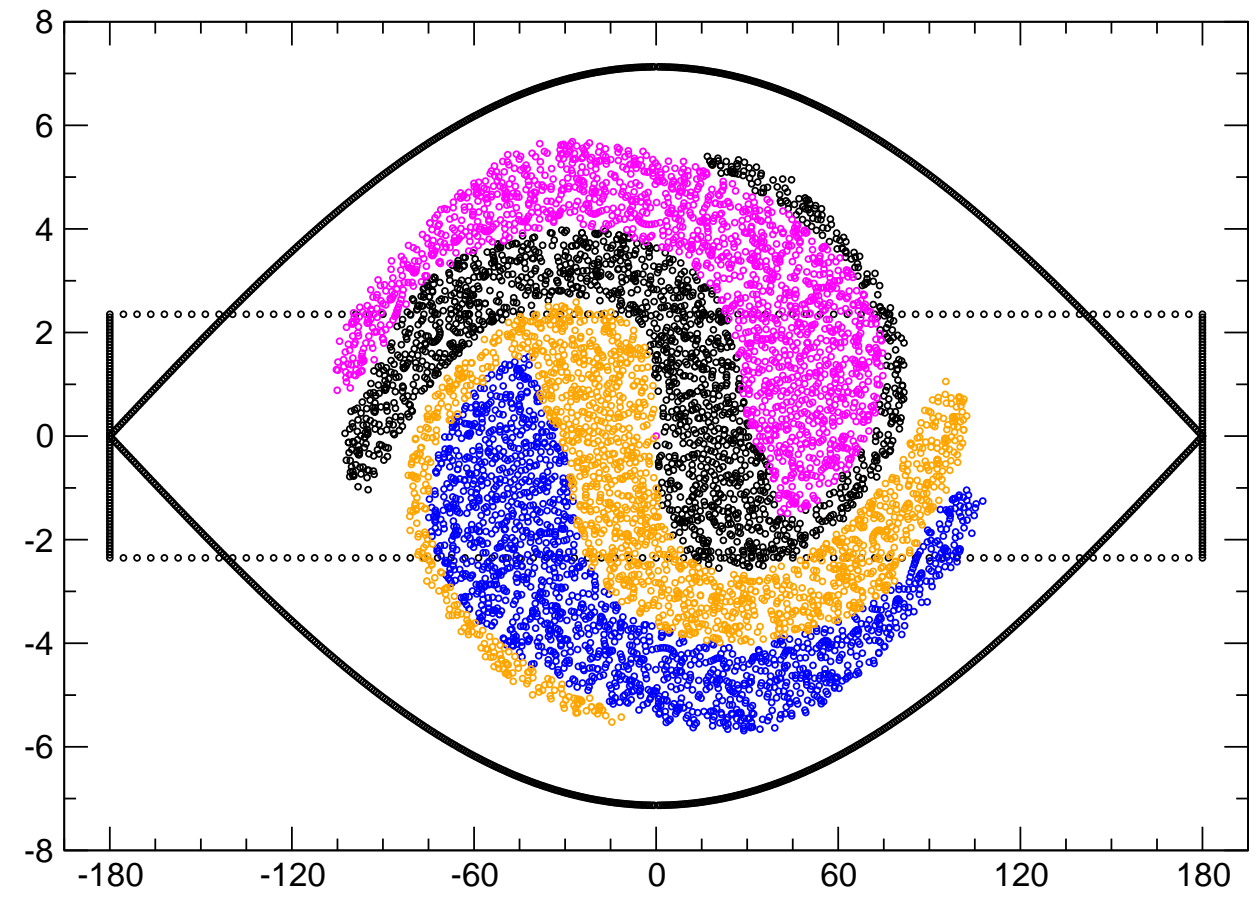

Figure 5: Completion of the 2-to- 1 merge. The harmonic 1 voltage is $V_{L}=$ $1.0 \mathrm{kV}$. The total 2 -to- 1 merge time is $T_{L}-T_{K}=7.5 \mathrm{~ms}$. The total 4 -to- 1 merge time is $T_{L}=15 \mathrm{~ms}$. The fractional momentum spread of the bunch is $\Delta p / p= \pm 0.00088$. The tails seen here make the effective emittance of the bunch larger than that of the initial 4 bunches. This is a result of the short total merging time of $15 \mathrm{~ms}$. In practice we are limited to this time in order to avoid introducing potentially harmful harmonics on the local power grid. Figure 6 shows the final bunch for the case in which the two merge times are doubled. The effective emittance in this case is noticeably reduced. 


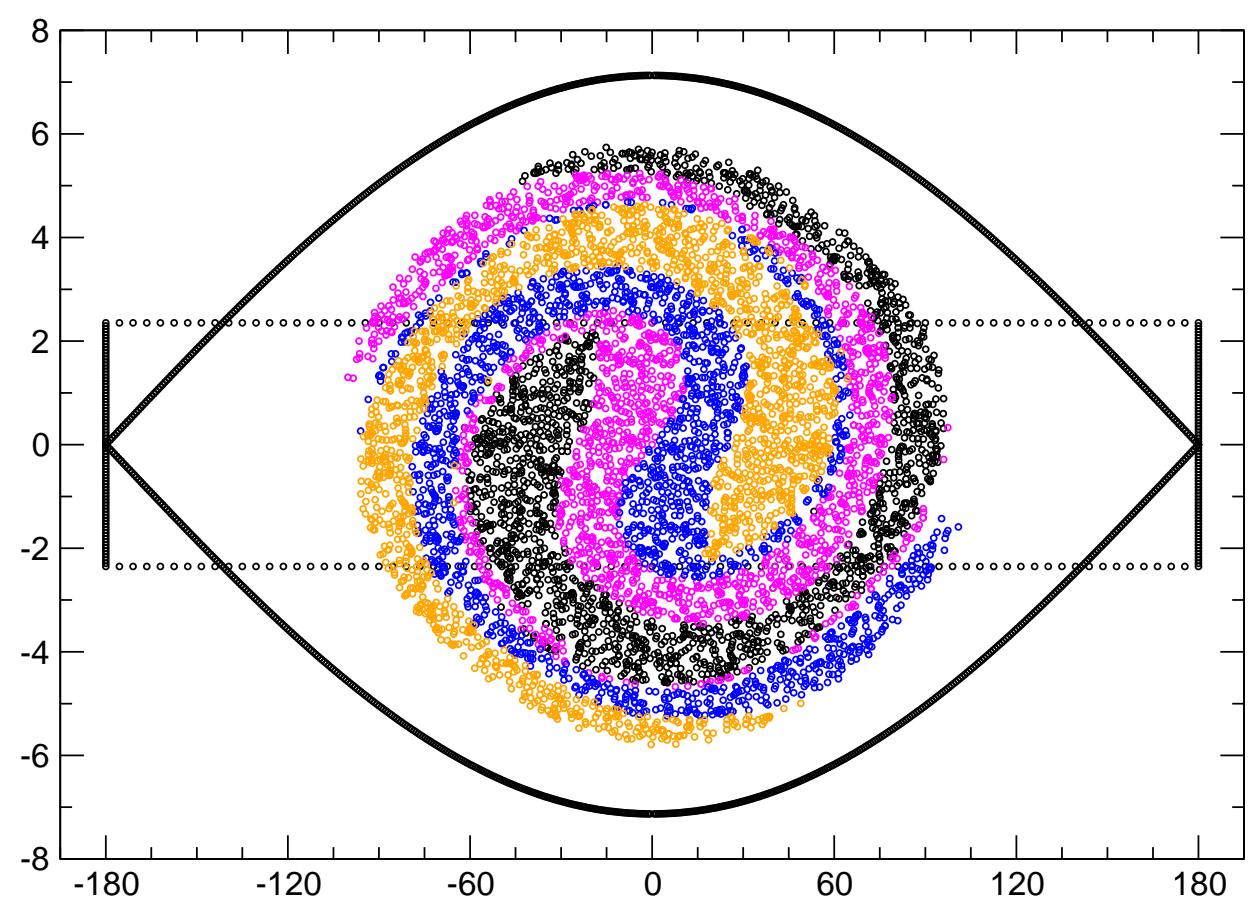

Figure 6: Same as Figure 5 but with merge times doubled. The effective emittance of the bunch is noticeably reduced. 


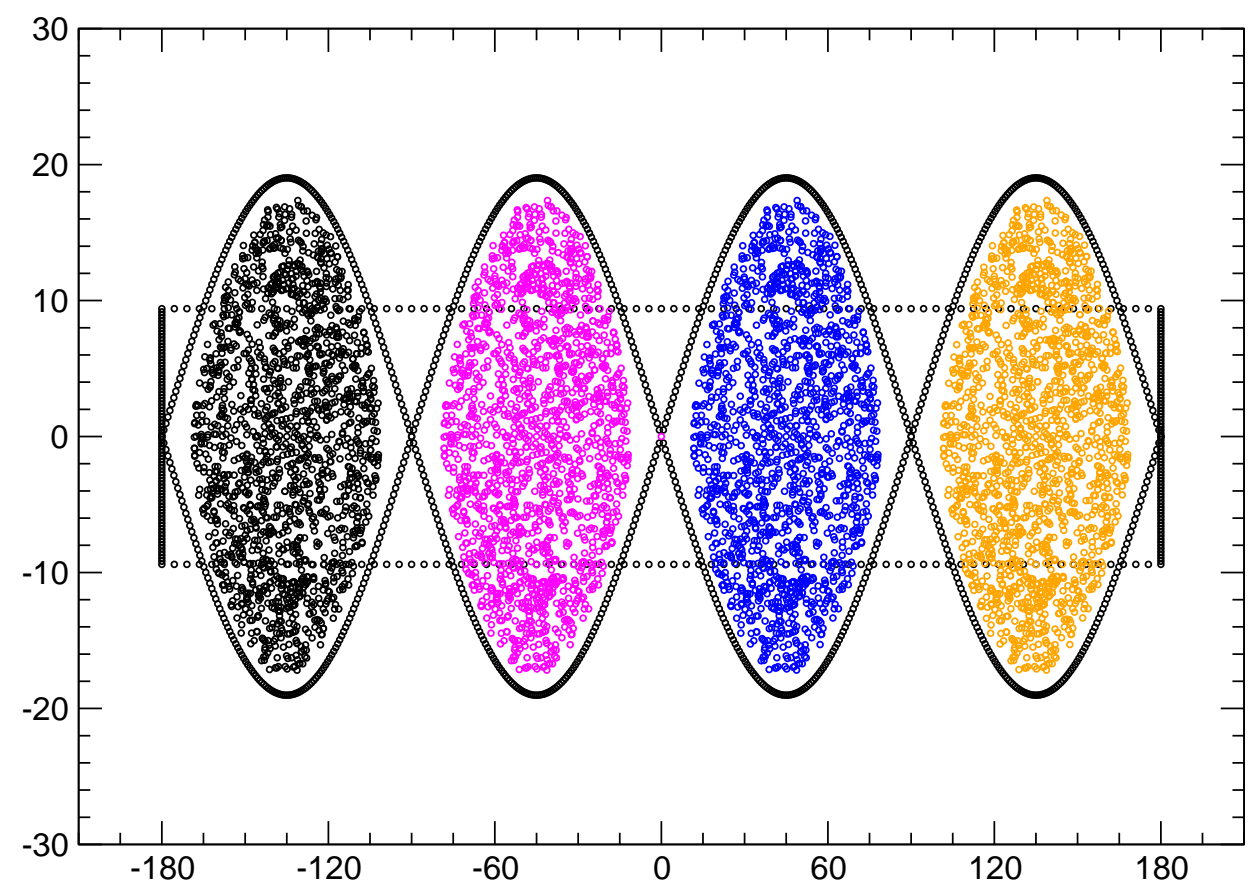

Figure 7: Four bunches of Au77+ ions in harmonic 16 buckets on the AGS injection porch. The harmonic 16 frequency is $16 f_{s}=2.61 \mathrm{MHz}$. The voltage is $V_{C}=40 \mathrm{kV}$. The black rectangle is the border of the uniform distribution used to make the bunches. The longitudinal emittance of the distribution is $0.60 \mathrm{eVs}$ per nucleon. One can see by inspection that the emittance of the 4 bunches is close to that of the initial distribution. The horizontal axis is the phase $\phi$ in degrees. The vertical axis is $W$ in units of eVs. 


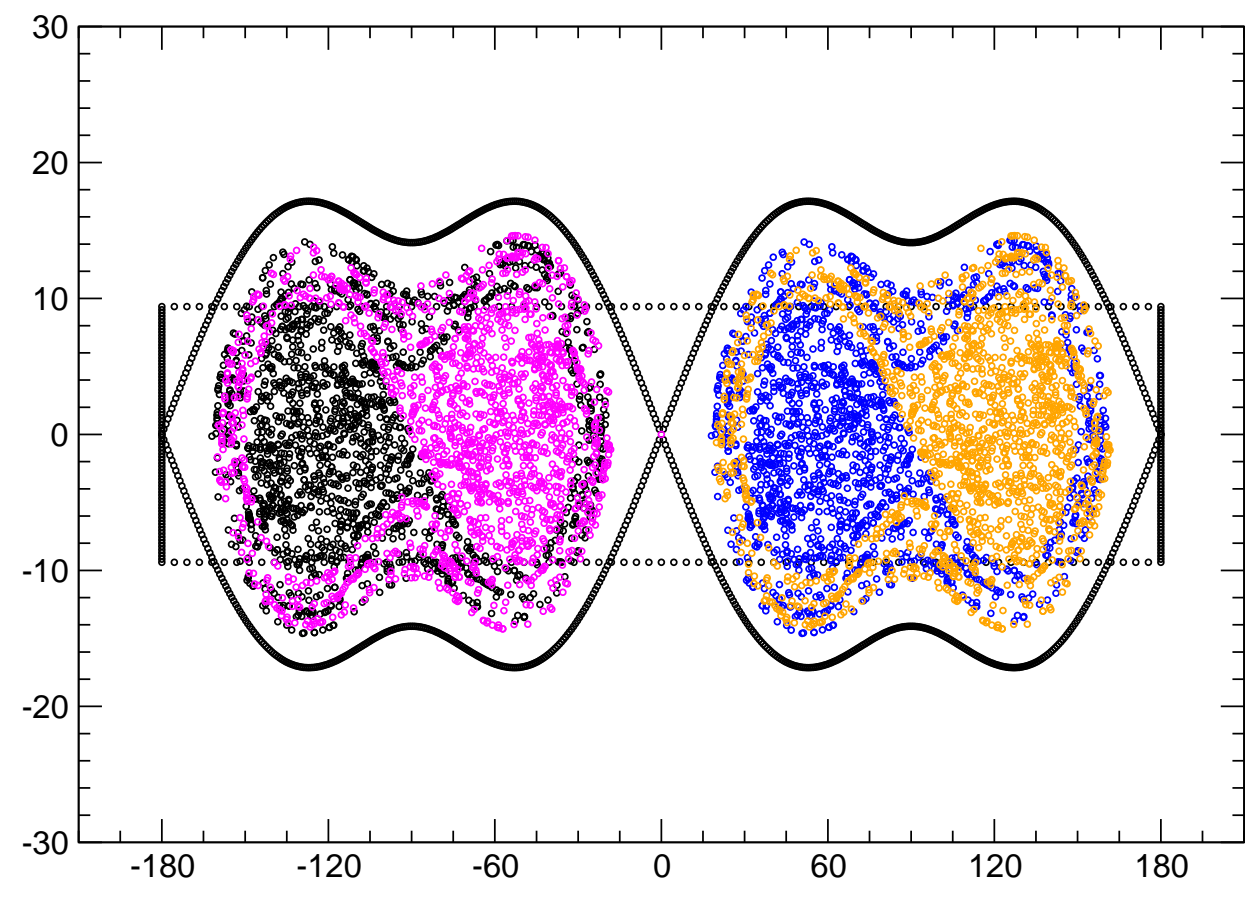

Figure 8: Halfway through the 4-to-2 merge of the bunches in Figure 7. Here the harmonic 16 voltage is $V_{C} / 2=20 \mathrm{kV}$. The harmonic 8 voltage is $V_{K} / 2=11 \mathrm{kV}$. The time from the start of the merge is $T_{K} / 2=15 / 2 \mathrm{~ms}$. 


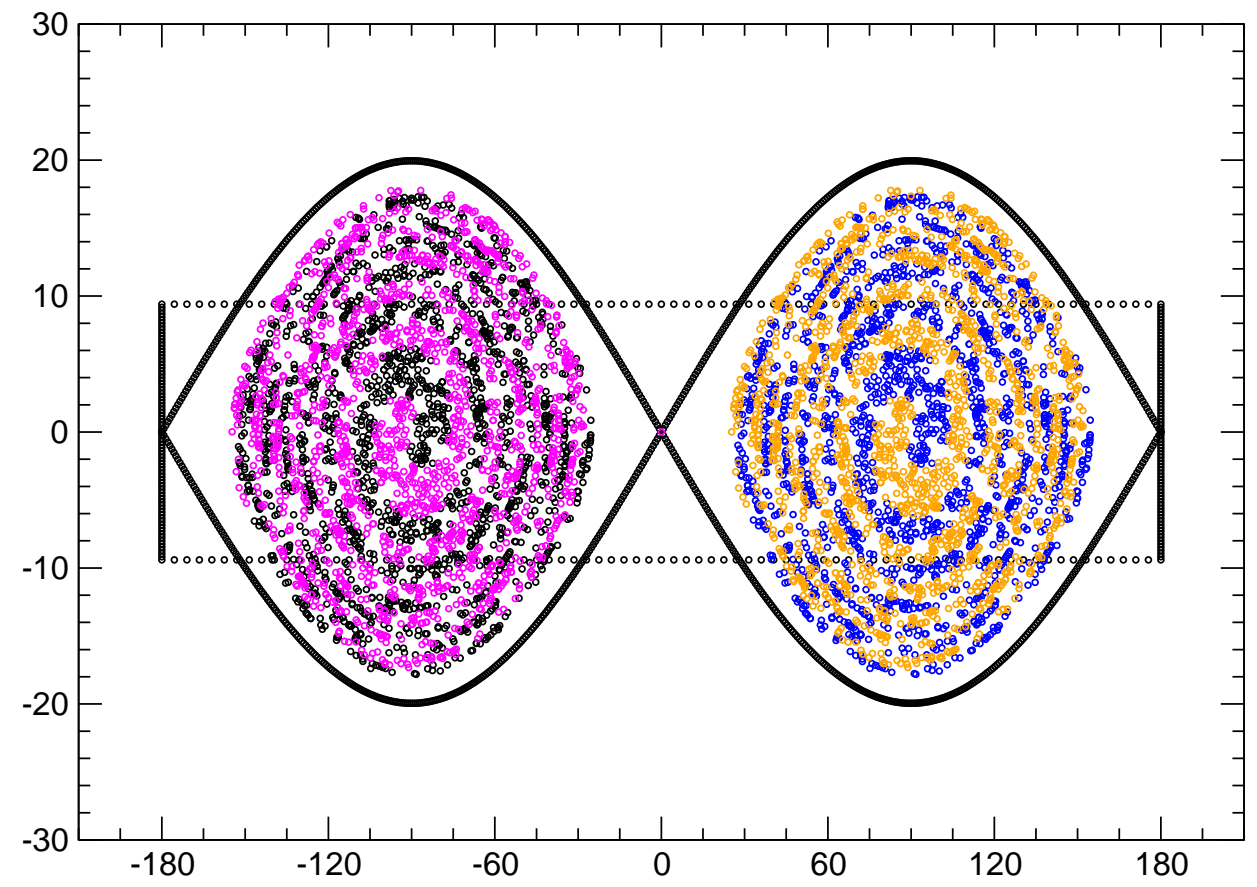

Figure 9: Completion of the 4-to-2 merge. Here the harmonic 8 voltage is $V_{K}=22 \mathrm{kV}$. The total time for the merge is $T_{K}=15 \mathrm{~ms}$. 


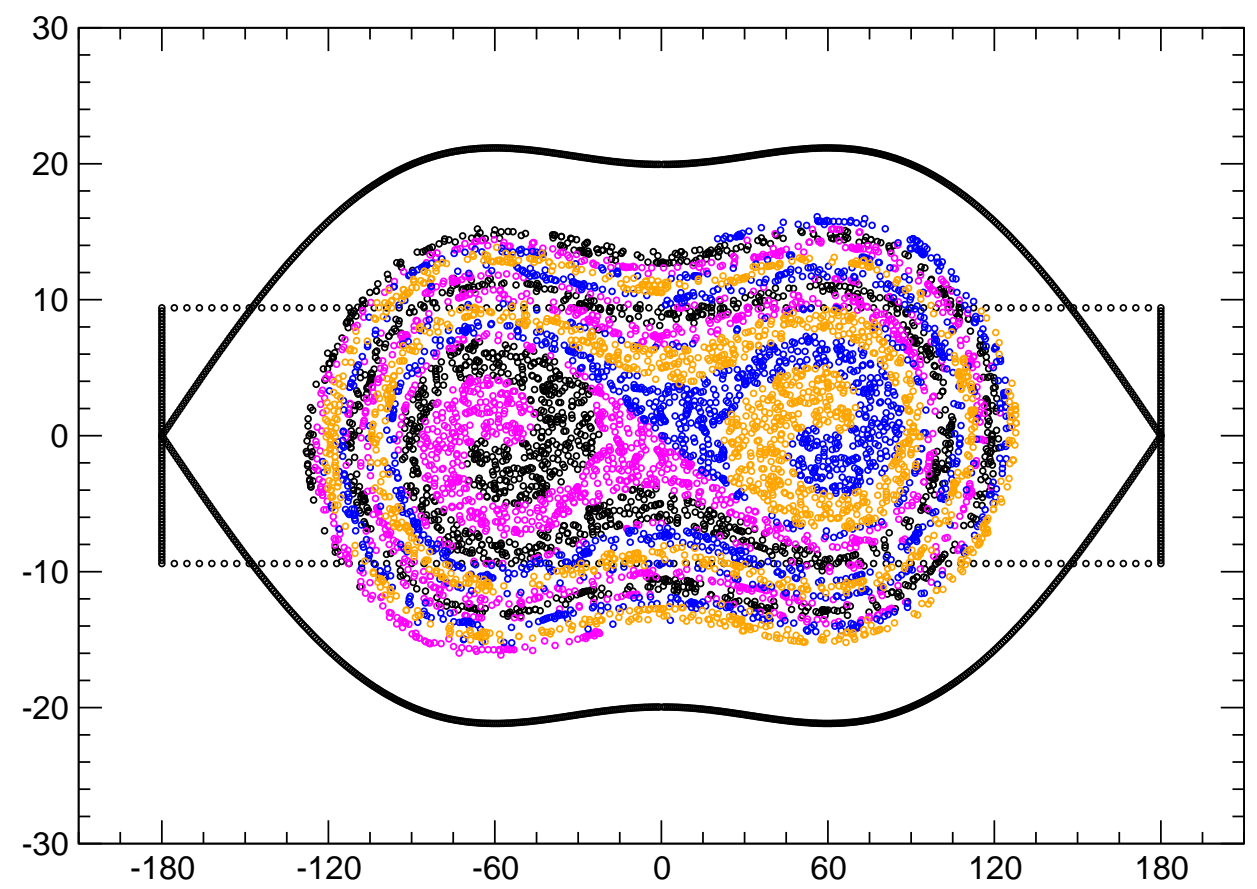

Figure 10: Halfway through the 2-to-1 merge of the bunches in Figure 9. Here the harmonic 8 voltage is $V_{K} / 2=11 \mathrm{kV}$. The harmonic 4 voltage is $V_{L} / 2=11 \mathrm{kV}$. The time from the start of the 2-to- 1 merge is $\left(T_{L}-T_{K}\right) / 2=$ $15 / 2 \mathrm{~ms}$. 


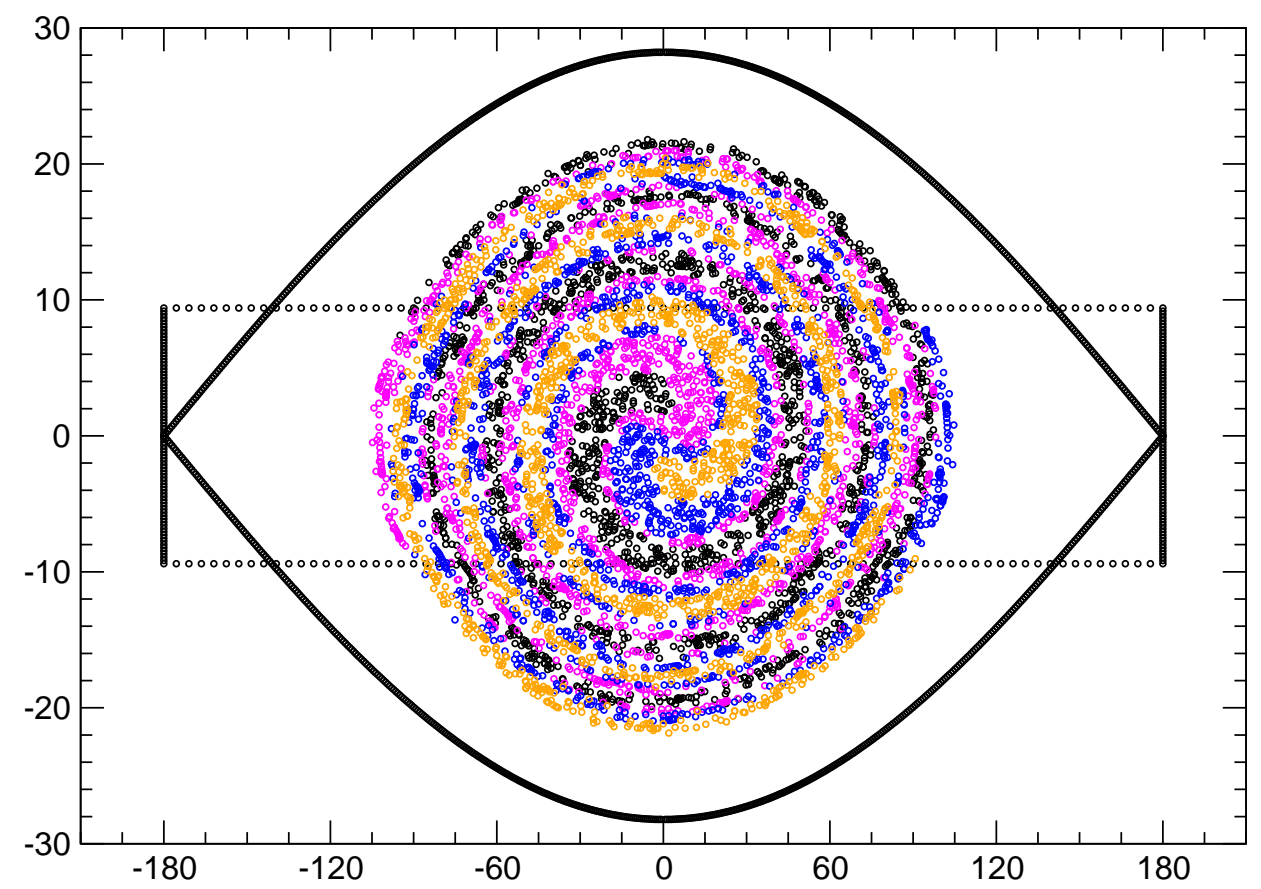

Figure 11: Completion of the 2-to- 1 merge. The harmonic 4 voltage is $V_{L}=22 \mathrm{kV}$. The total 2-to- 1 merge time is $T_{L}-T_{K}=15 \mathrm{~ms}$. The total 4-to- 1 merge time is $T_{L}=30 \mathrm{~ms}$. The fractional momentum spread of the bunch is $\Delta p / p= \pm 0.0023$. One can see by inspection that the emittance of the bunch is close to that $(0.60 \mathrm{eV}$ s per nucleon $)$ of the initial distribution of unbunched beam. It is easily held in the harmonic 4 bucket. 


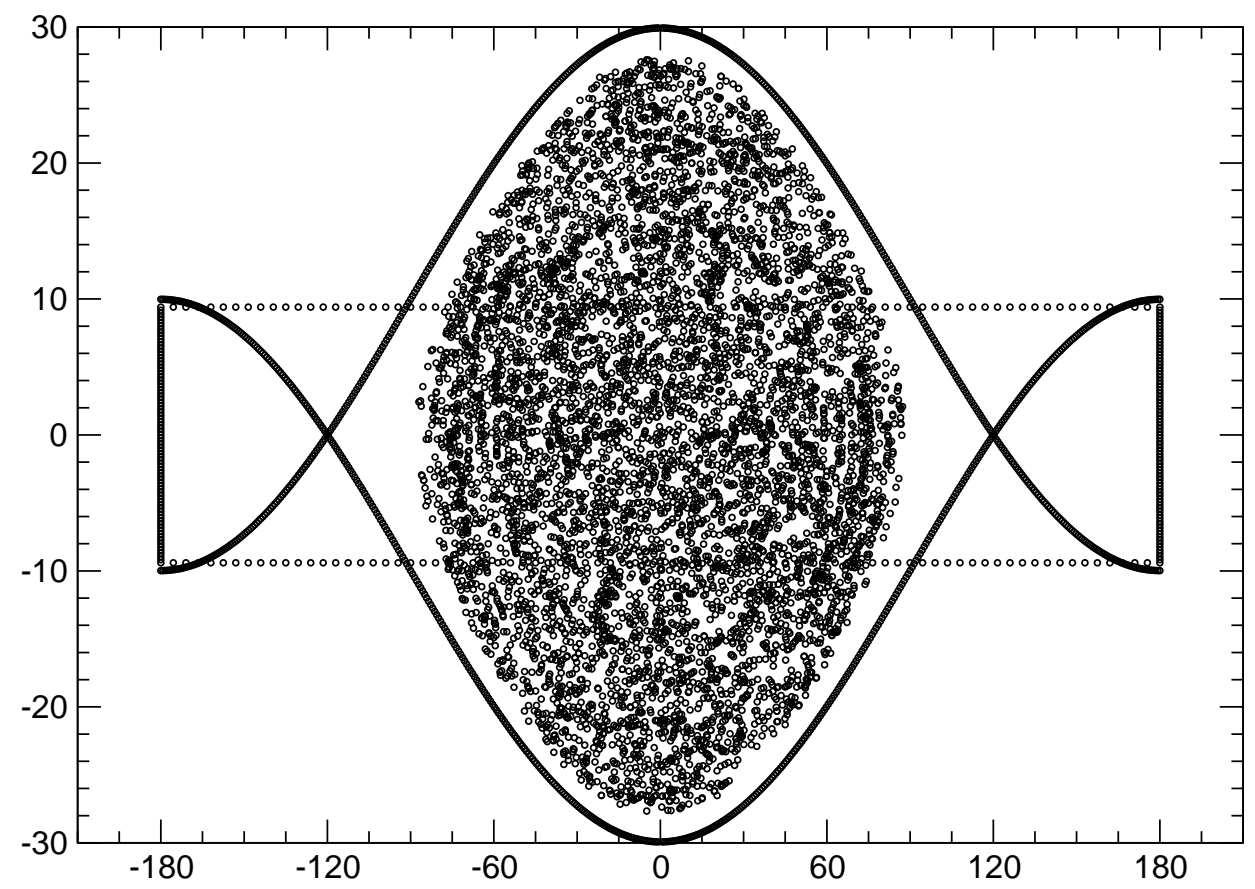

Figure 12: Here the merged bunch of Figure $\mathbf{1 1}$ has been squeezed by bringing on the harmonic 8 voltage. The voltage is raised from zero to $V_{K}=22 \mathrm{kV}$ in $20 \mathrm{~ms}$. The harmonic 4 voltage is held constant at $V_{L}=22$ $\mathrm{kV}$. The unstable fixed point phases are $\phi_{u}= \pm 120$ degrees. 


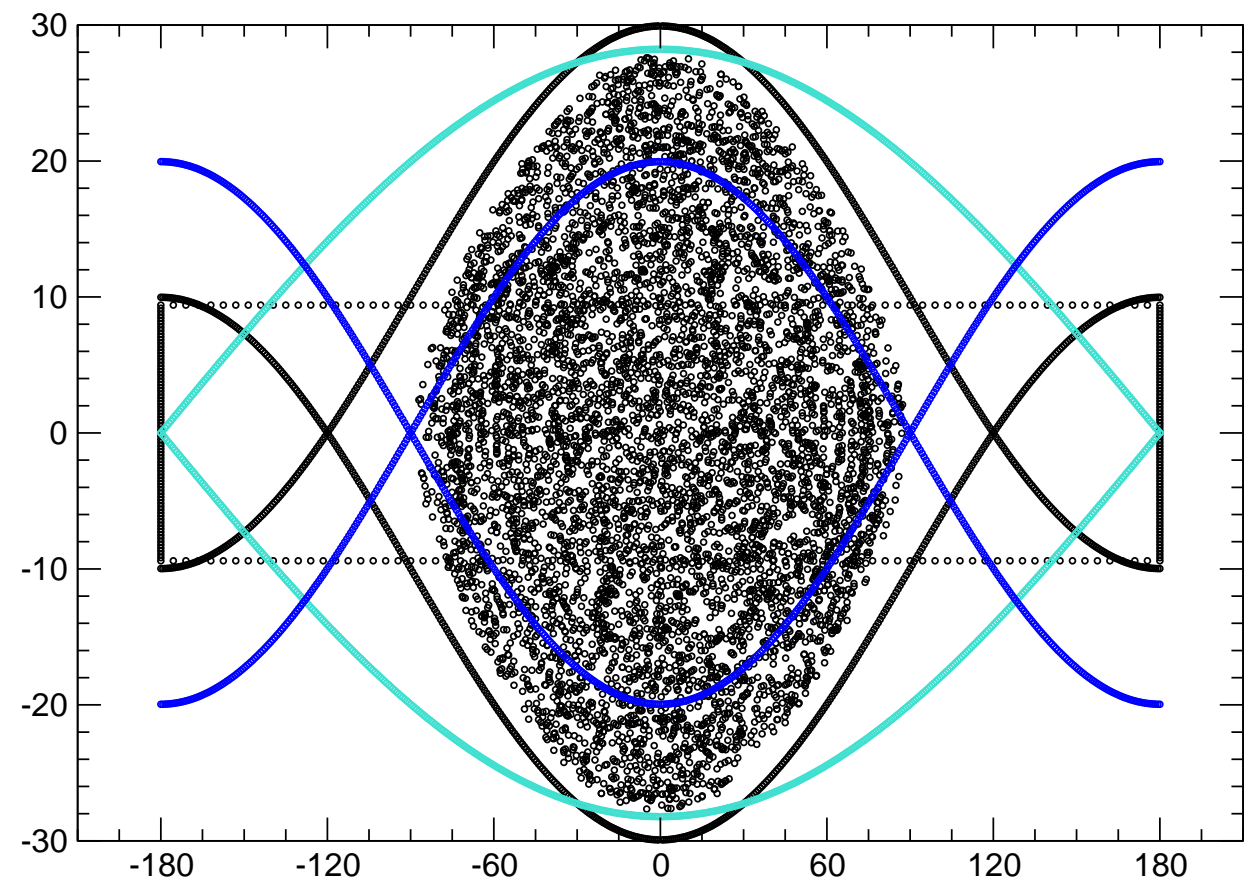

Figure 13: Same as Figure 12 but with the harmonic 4 and 8 buckets superimposed. These act together with voltages $V_{L}=22 \mathrm{kV}$ and $V_{K}=22$ $\mathrm{kV}$ to make the black separatrix. The harmonic 8 bucket acting by itself is not large enough to contain the bunch. 


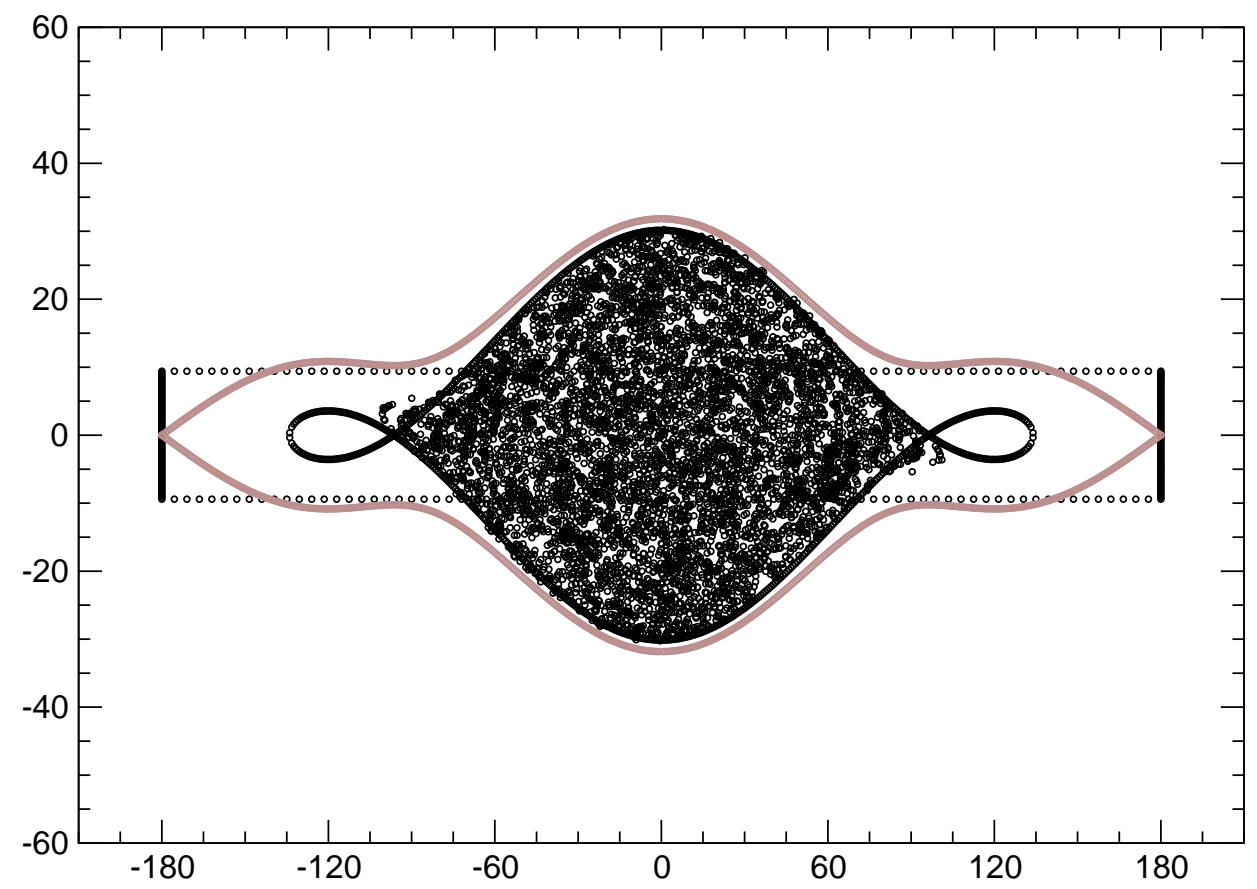

Figure 14: Here the bunch of Figure $\mathbf{1 2}$ has been squeezed by bringing on the harmonic 12 voltage. The voltage is raised from zero to $V_{A}=18 \mathrm{kV}$ in $1 \mathrm{~ms}$. The harmonic 4 and 8 voltages are held constant at $V_{L}=22 \mathrm{kV}$ and $V_{K}=22 \mathrm{kV}$ respectively. The unstable fixed point phases are $\phi_{u}= \pm 96.4$ and $\phi_{u}= \pm 180$ degrees. 


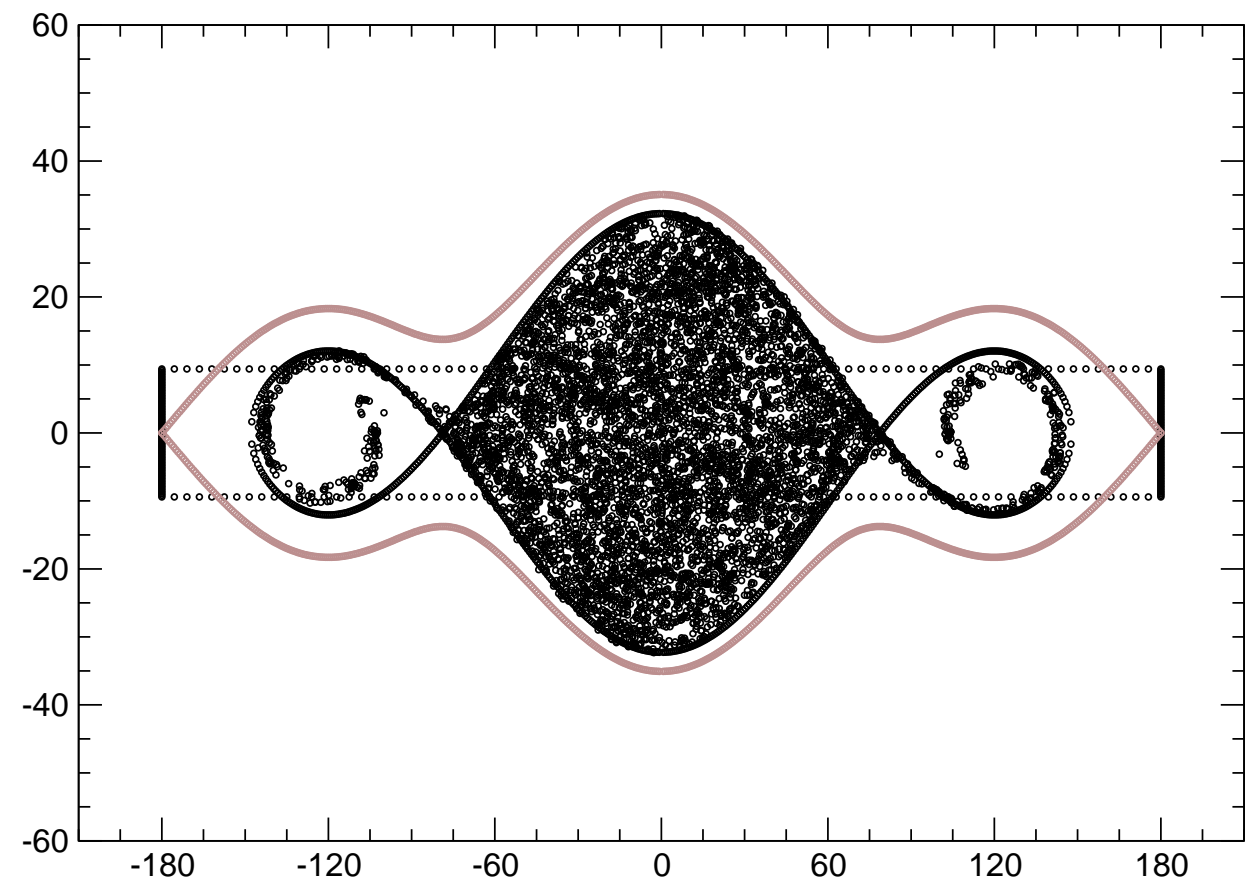

Figure 15: Here, with the harmonic 4 and 8 voltages held at $V_{L}=22 \mathrm{kV}$ and $V_{K}=22 \mathrm{kV}$ respectively, the harmonic 12 voltage has been raised from zero to $V_{A}=36 \mathrm{kV}$ in $2 \mathrm{~ms}$. The unstable fixed point phases are $\phi_{u}= \pm 78.8$ and $\phi_{u}= \pm 180$ degrees. 


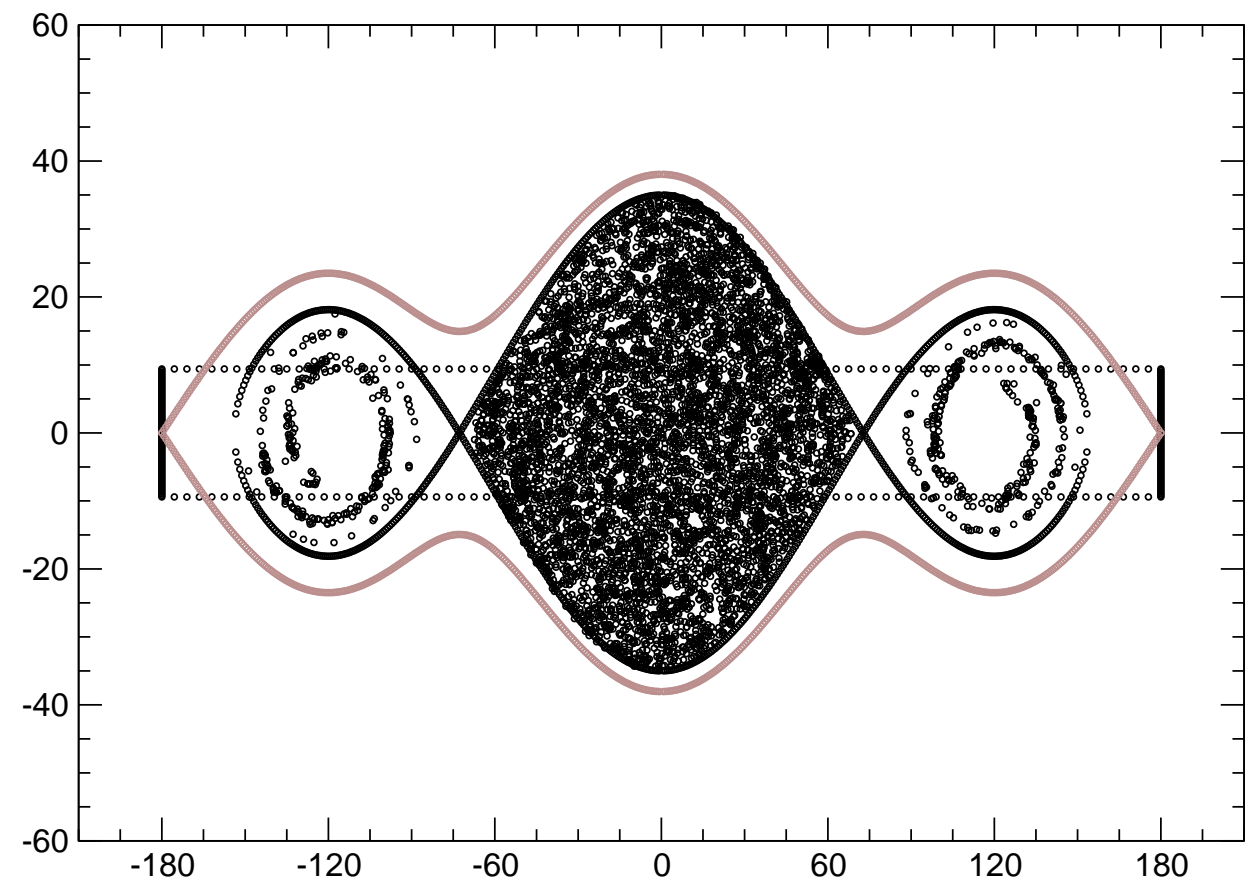

Figure 16: Here, with the harmonic 4 and 8 voltages held at $V_{L}=22 \mathrm{kV}$ and $V_{K}=22 \mathrm{kV}$ respectively, the harmonic 12 voltage has been raised from zero to $V_{A}=54 \mathrm{kV}$ in $3 \mathrm{~ms}$. The unstable fixed point phases are $\phi_{u}= \pm 72.8$ and $\phi_{u}= \pm 180$ degrees. 


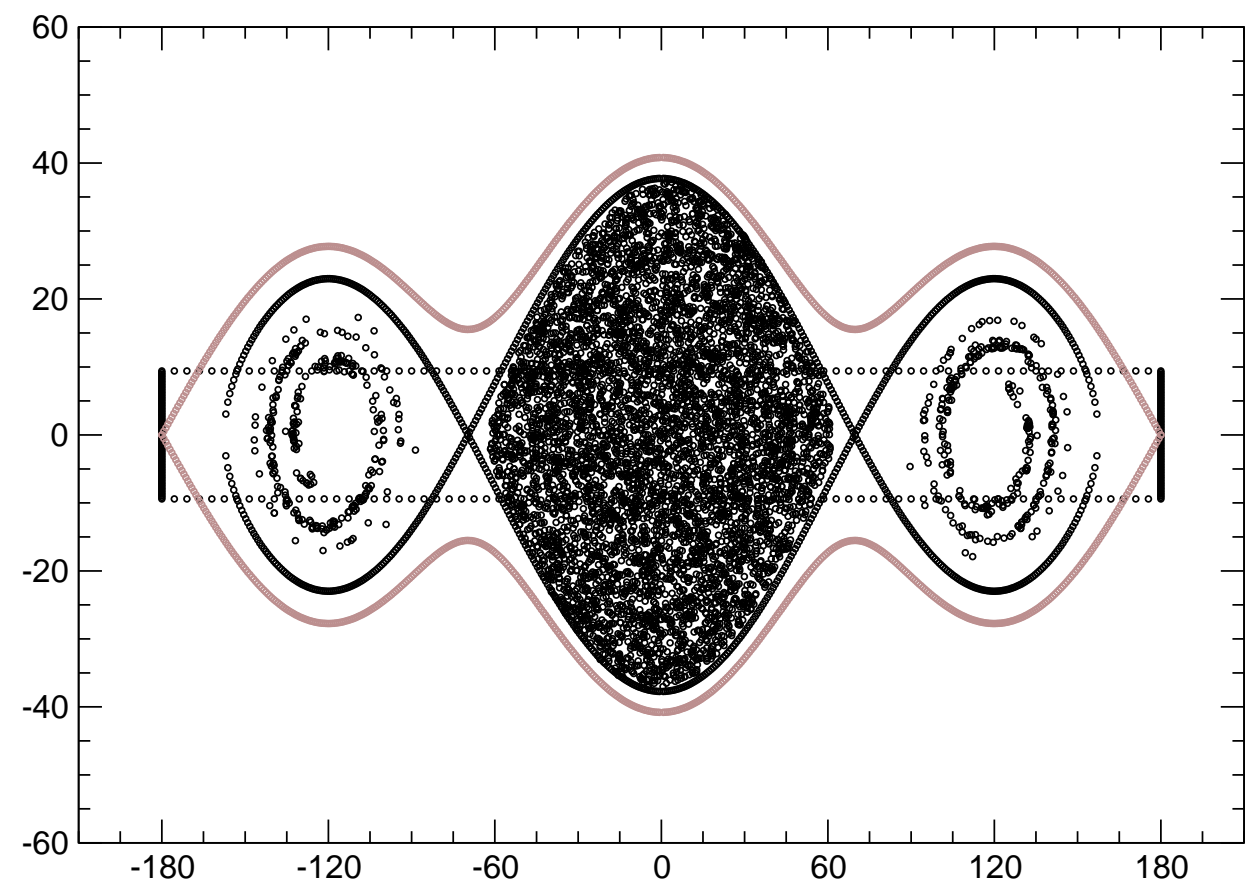

Figure 17: Here, with the harmonic 4 and 8 voltages held at $V_{L}=22 \mathrm{kV}$ and $V_{K}=22 \mathrm{kV}$ respectively, the harmonic 12 voltage has been raised from zero to $V_{A}=72 \mathrm{kV}$ in $4 \mathrm{~ms}$. The unstable fixed point phases are $\phi_{u}= \pm 69.7$ and $\phi_{u}= \pm 180$ degrees. 


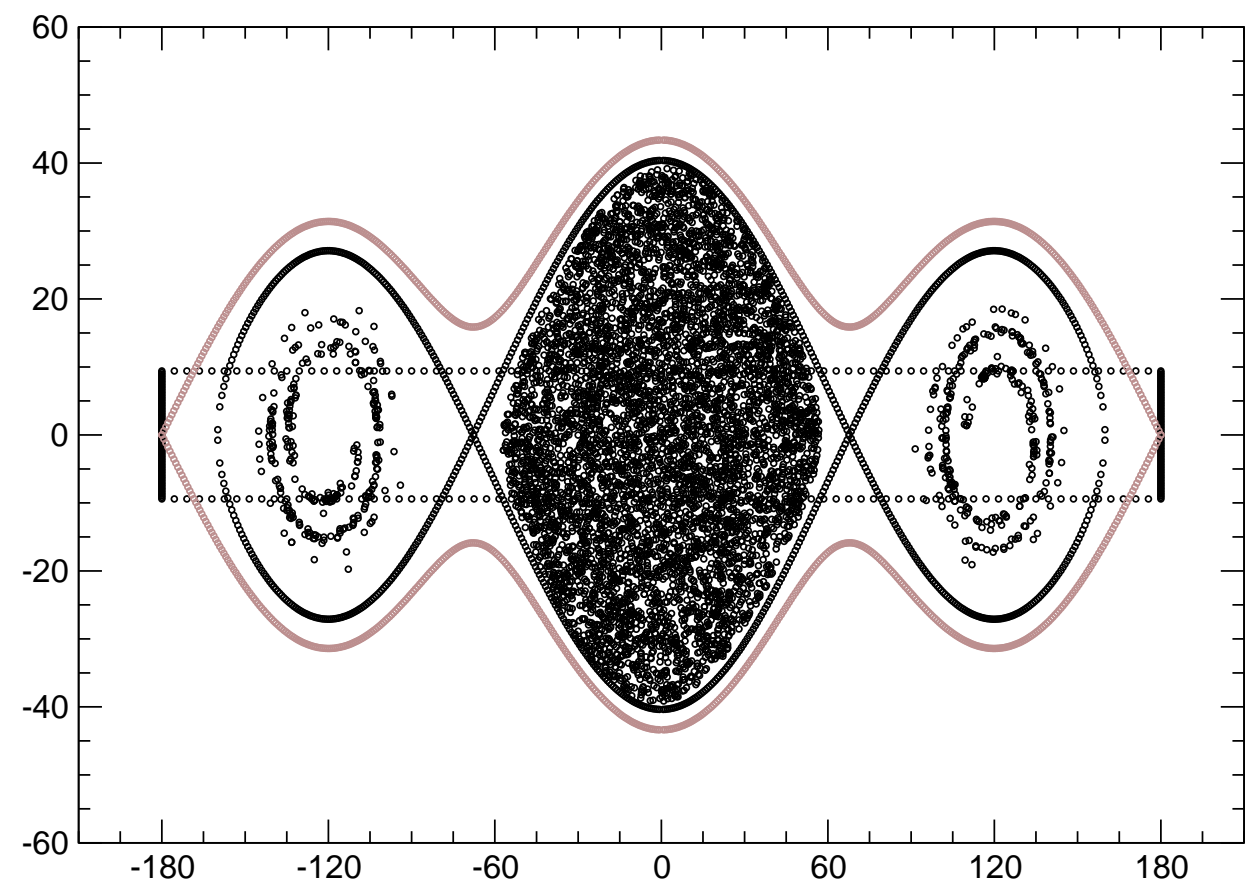

Figure 18: Here, with the harmonic 4 and 8 voltages held at $V_{L}=22 \mathrm{kV}$ and $V_{K}=22 \mathrm{kV}$ respectively, the harmonic 12 voltage has been raised from zero to $V_{A}=90 \mathrm{kV}$ in $5 \mathrm{~ms}$. The unstable fixed point phases are $\phi_{u}= \pm 67.8$ and $\phi_{u}= \pm 180$ degrees. 


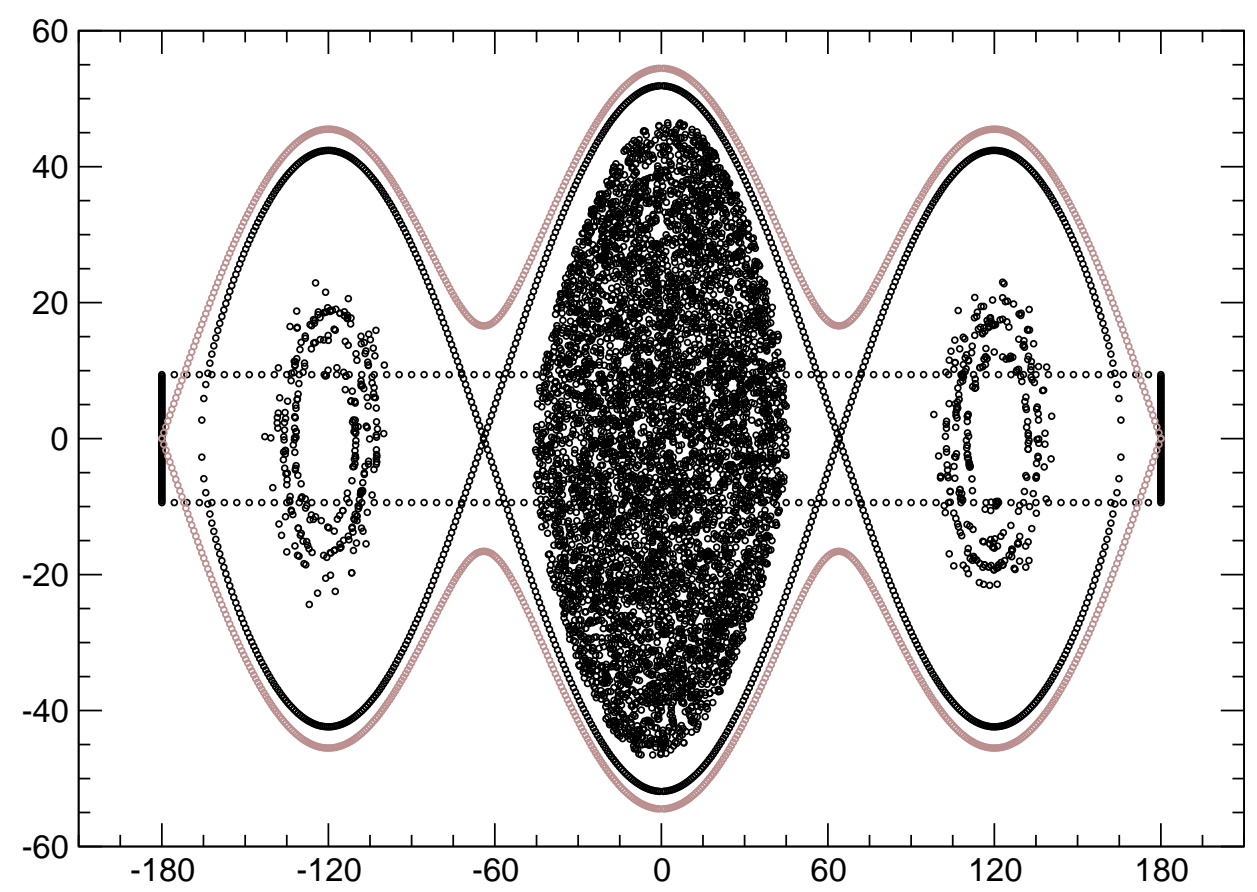

Figure 19: Here, with the harmonic 4 and 8 voltages held at $V_{L}=22 \mathrm{kV}$ and $V_{K}=22 \mathrm{kV}$ respectively, the harmonic 12 voltage has been raised from zero to $V_{A}=180 \mathrm{kV}$ in $10 \mathrm{~ms}$. The unstable fixed point phases are $\phi_{u}= \pm 64.0$ and $\phi_{u}= \pm 180$ degrees. The fractional momentum spread of the central bunch is $\Delta p / p= \pm 0.00484$. The fraction of Au77+ ions in the outer buckets is 0.089 . 


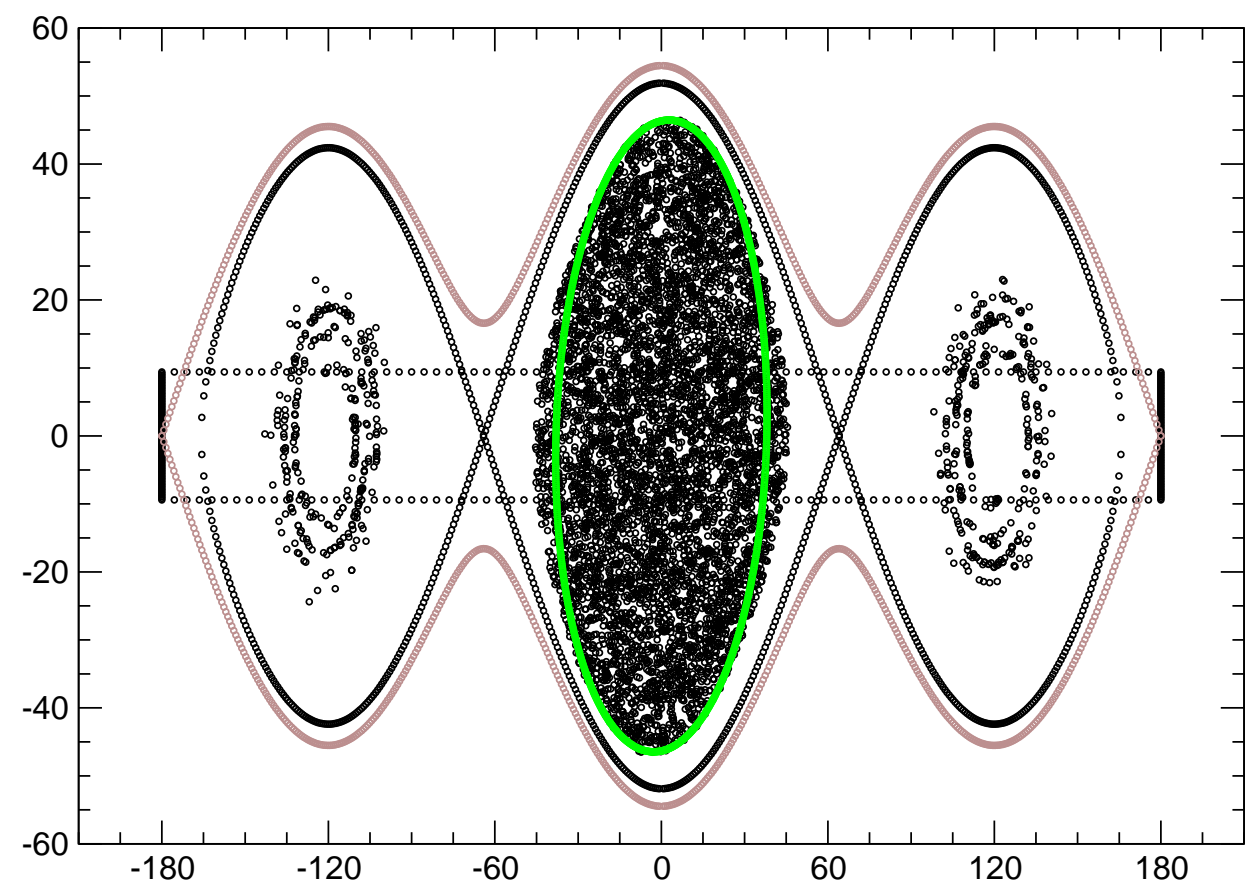

Figure 20: Same as Figure 19 but with green ellipse added. The bunch and ellipse are rotated slightly clockwise with respect to the vertical axis. This is a feature of the motion generated by (38) and (39) and is discussed in the Appendix. 


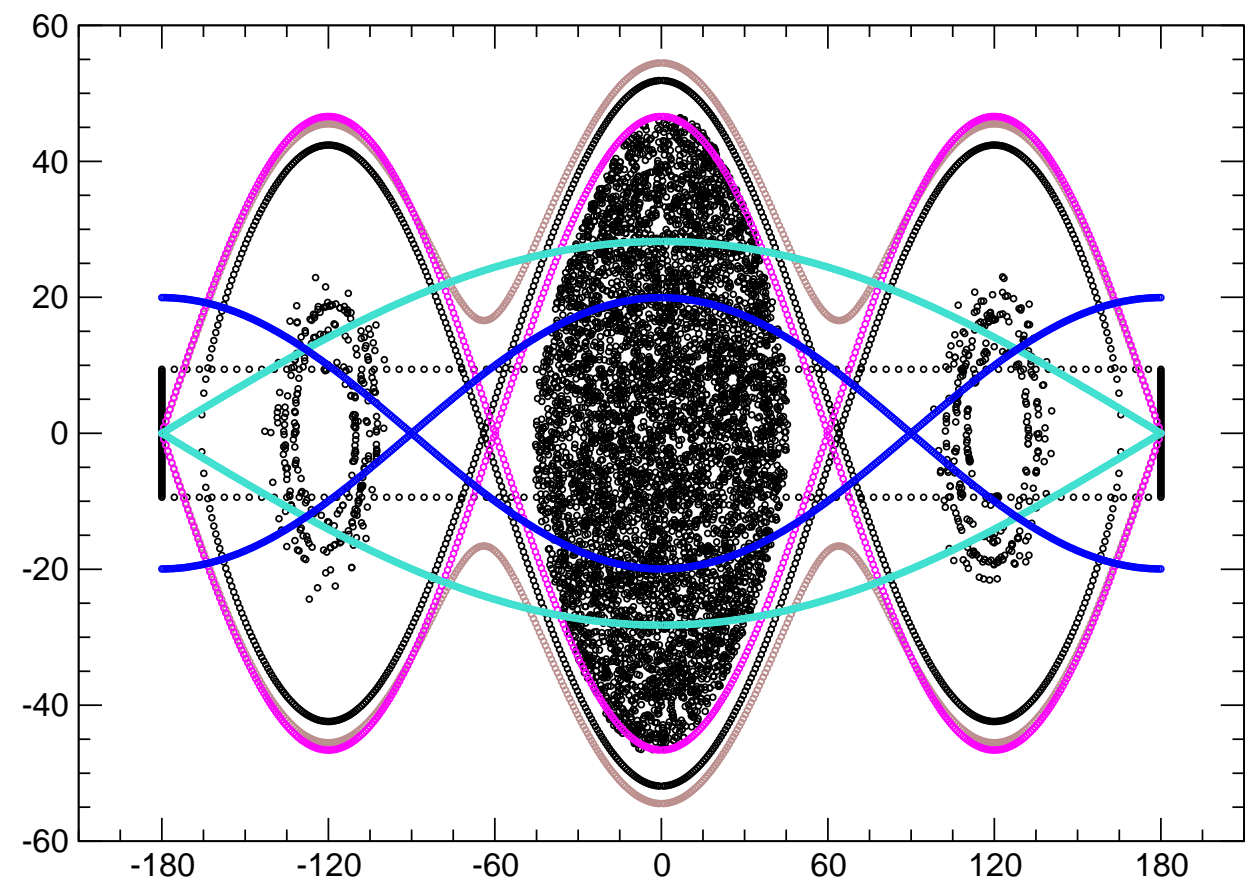

Figure 21: Same as Figure 19 but with the harmonic 4, 8, and 12 buckets superimposed. These act together with voltages $V_{L}=22 \mathrm{kV}, V_{K}=22 \mathrm{kV}$, and $V_{A}=180 \mathrm{kV}$ to make the black and brown separatrices. 


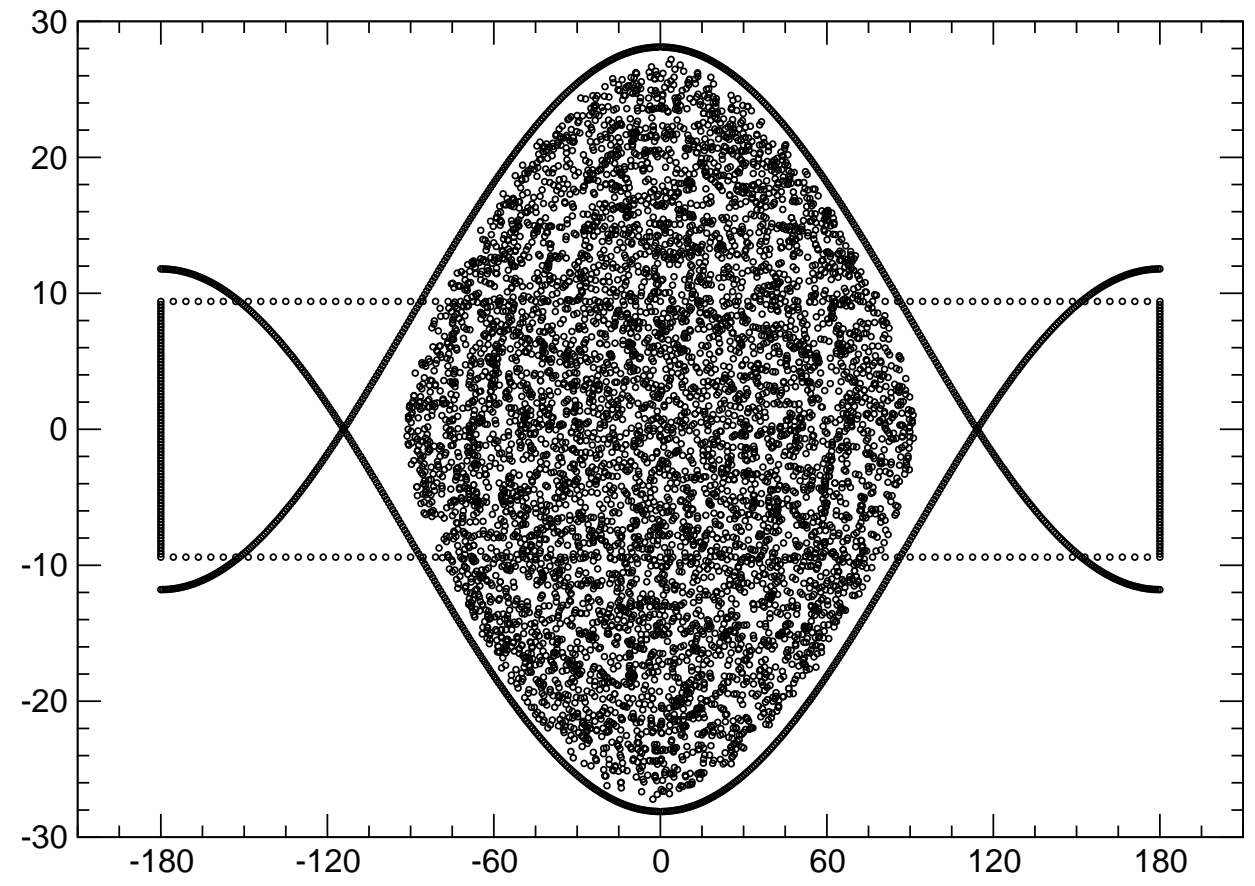

Figure 22: Here the simulation that produced Figures 7 through 19 has been carried out with the maximum harmonic 4 voltage reduced to $V_{L}=18$ $\mathrm{kV}$. The unstable fixed point phases are $\phi_{u}= \pm 114$ degrees. 


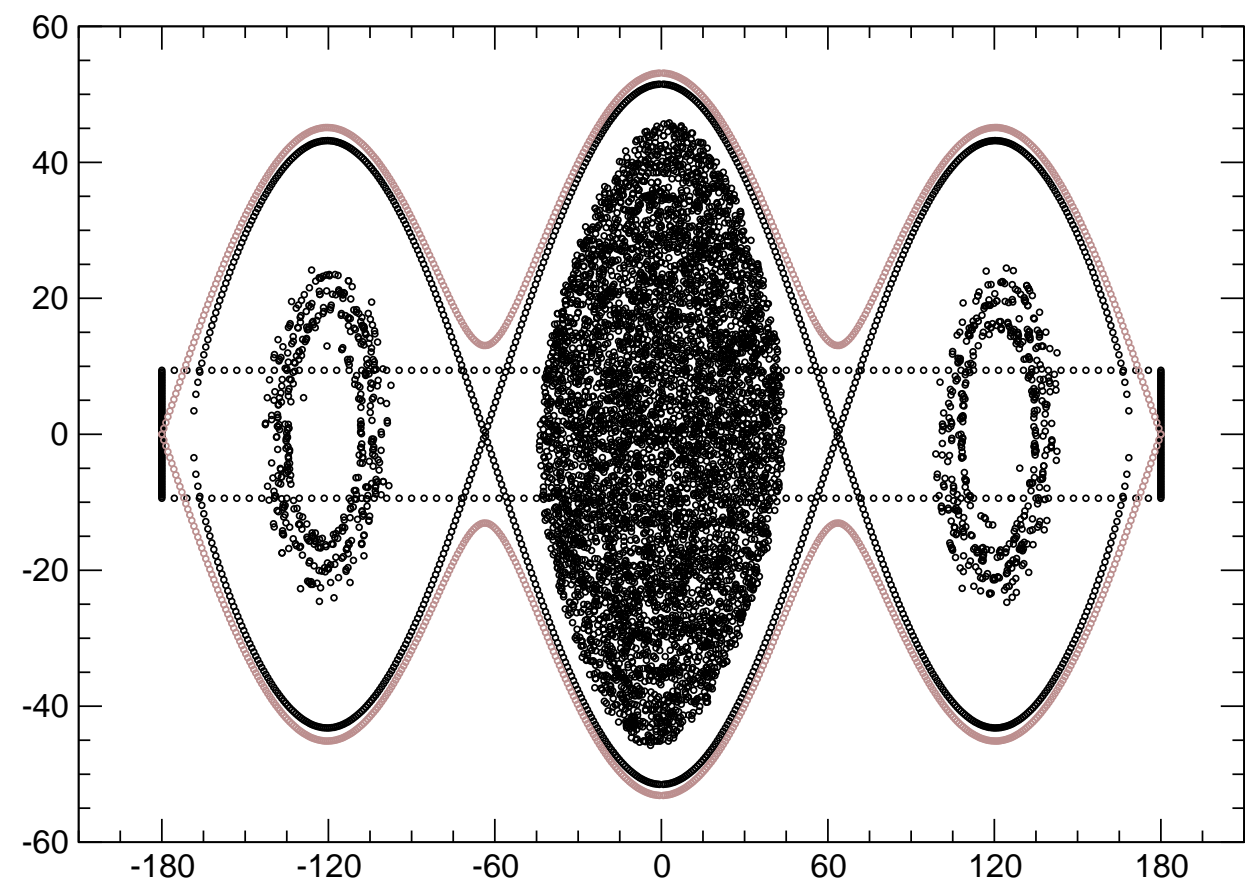

Figure 23: Here as in Figure 22 the simulation has been carried out with the maximum harmonic 4 voltage reduced to $V_{L}=18 \mathrm{kV}$. The unstable fixed point phases are $\phi_{u}= \pm 63.6$ and $\phi_{u}= \pm 180$ degrees. The fractional momentum spread of the central bunch is $\Delta p / p= \pm 0.00477$. The fraction of Au77+ ions in the outer buckets has increased to 0.138 . 


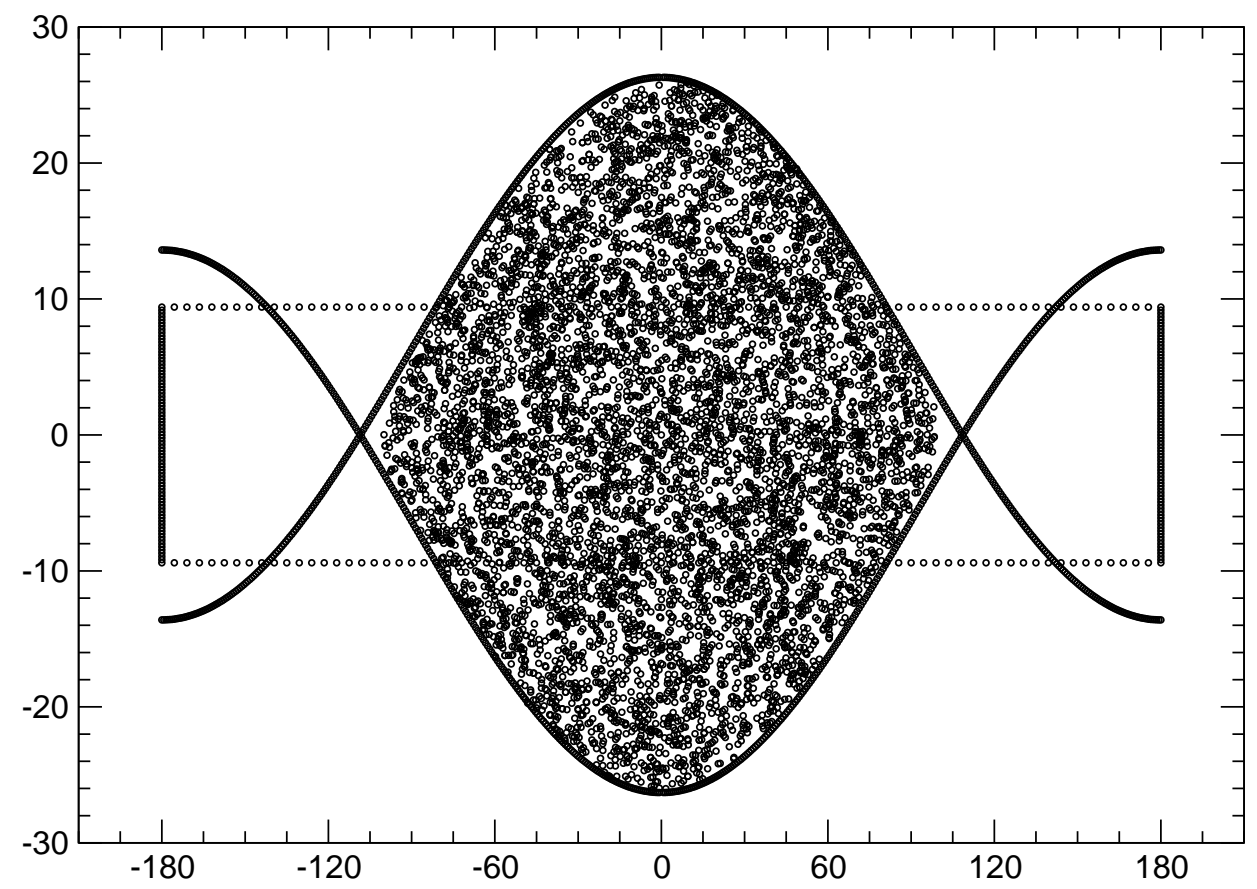

Figure 24: Here the simulation that produced Figures 7 through 19 has been carried out with the maximum harmonic 4 voltage reduced further to $V_{L}=14 \mathrm{kV}$. The bucket is now just large enough to contain the bunch. The unstable fixed point phases are $\phi_{u}= \pm 109$ degrees. 


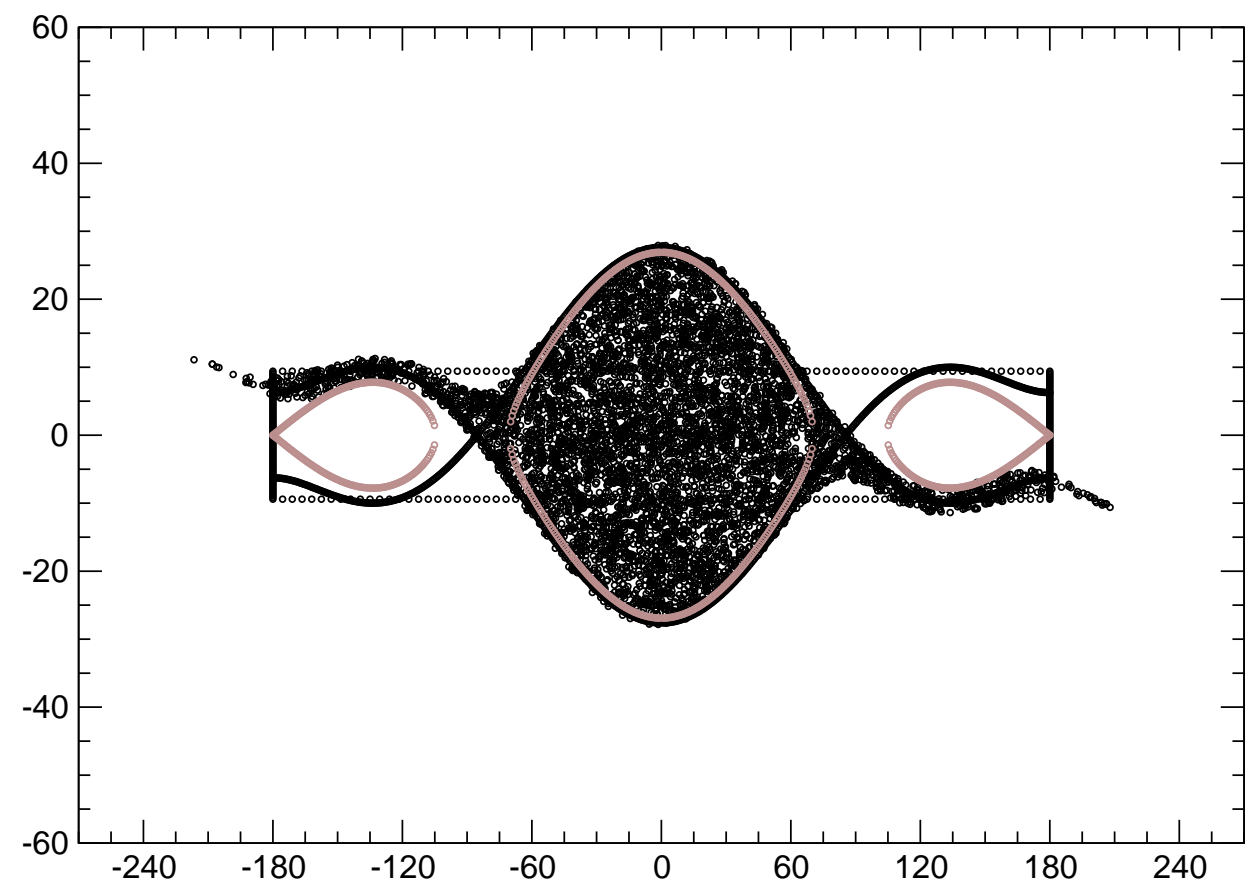

Figure 25: Here the bunch of Figure $\mathbf{2 4}$ has been squeezed by bringing on the harmonic 12 voltage. The voltage is raised from zero to $V_{A}=18 \mathrm{kV}$ in $1 \mathrm{~ms}$. The harmonic 4 and 8 voltages are held constant at $V_{L}=14 \mathrm{kV}$ and $V_{K}=22 \mathrm{kV}$ respectively. The unstable fixed point phases are $\phi_{u}= \pm 85.4$ and $\phi_{u}= \pm 180$ degrees. 


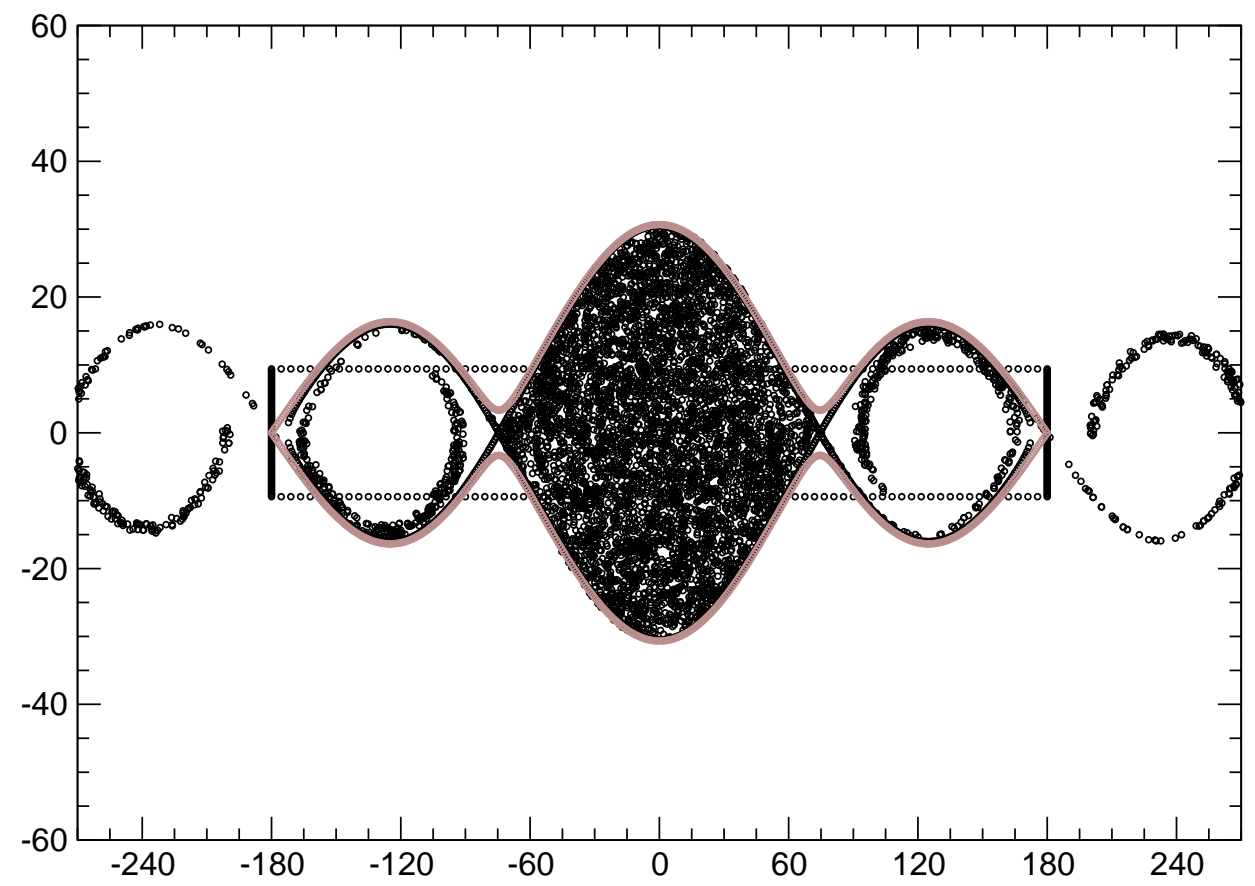

Figure 26: Here, with the harmonic 4 and 8 voltages held at $V_{L}=14 \mathrm{kV}$ and $V_{K}=22 \mathrm{kV}$ respectively, the harmonic 12 voltage has been raised from zero to $V_{A}=36 \mathrm{kV}$ in $2 \mathrm{~ms}$. The unstable fixed point phases are $\phi_{u}= \pm 74.5$ and $\phi_{u}= \pm 180$ degrees. 


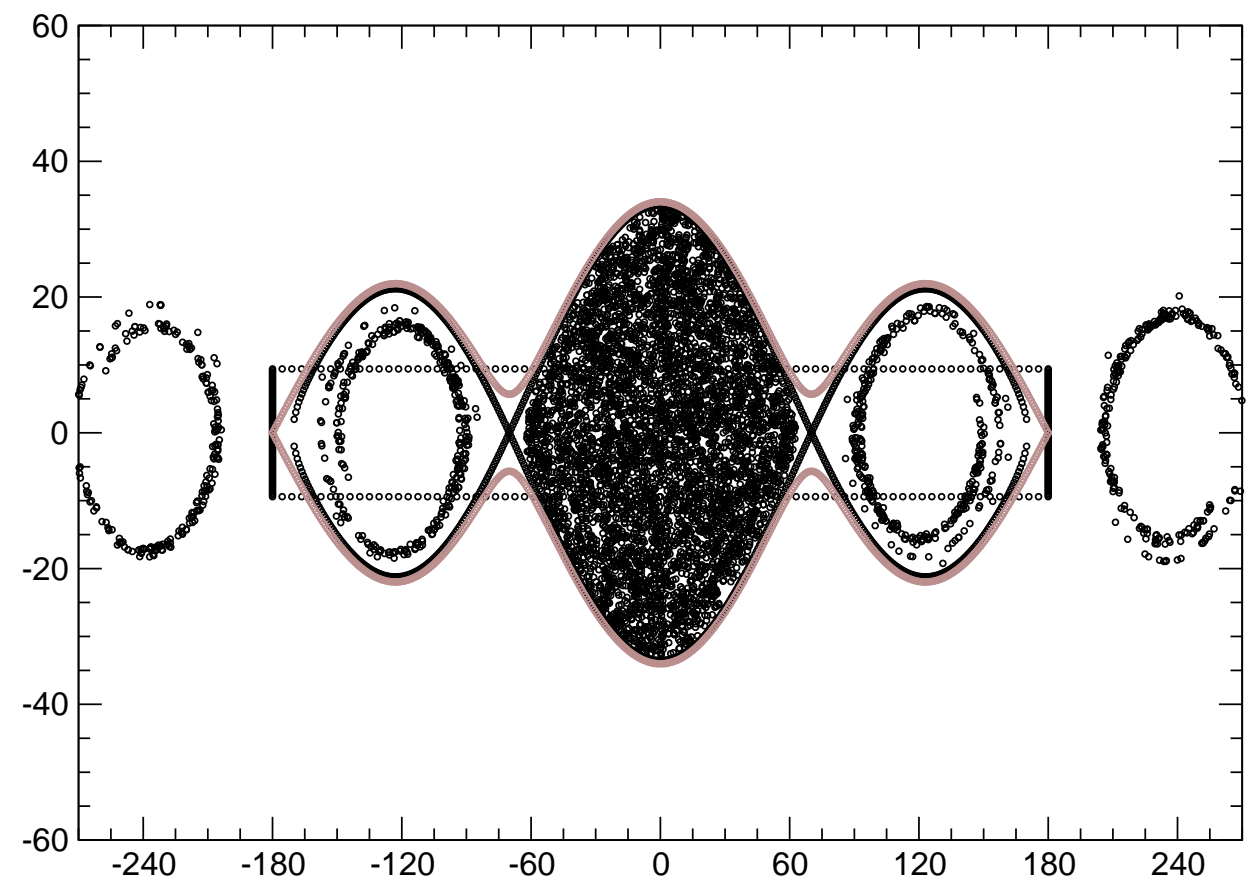

Figure 27: Here, with the harmonic 4 and 8 voltages held at $V_{L}=14 \mathrm{kV}$ and $V_{K}=22 \mathrm{kV}$ respectively, the harmonic 12 voltage has been raised from zero to $V_{A}=54 \mathrm{kV}$ in $3 \mathrm{~ms}$. The unstable fixed point phases are $\phi_{u}= \pm 70.1$ and $\phi_{u}= \pm 180$ degrees. 


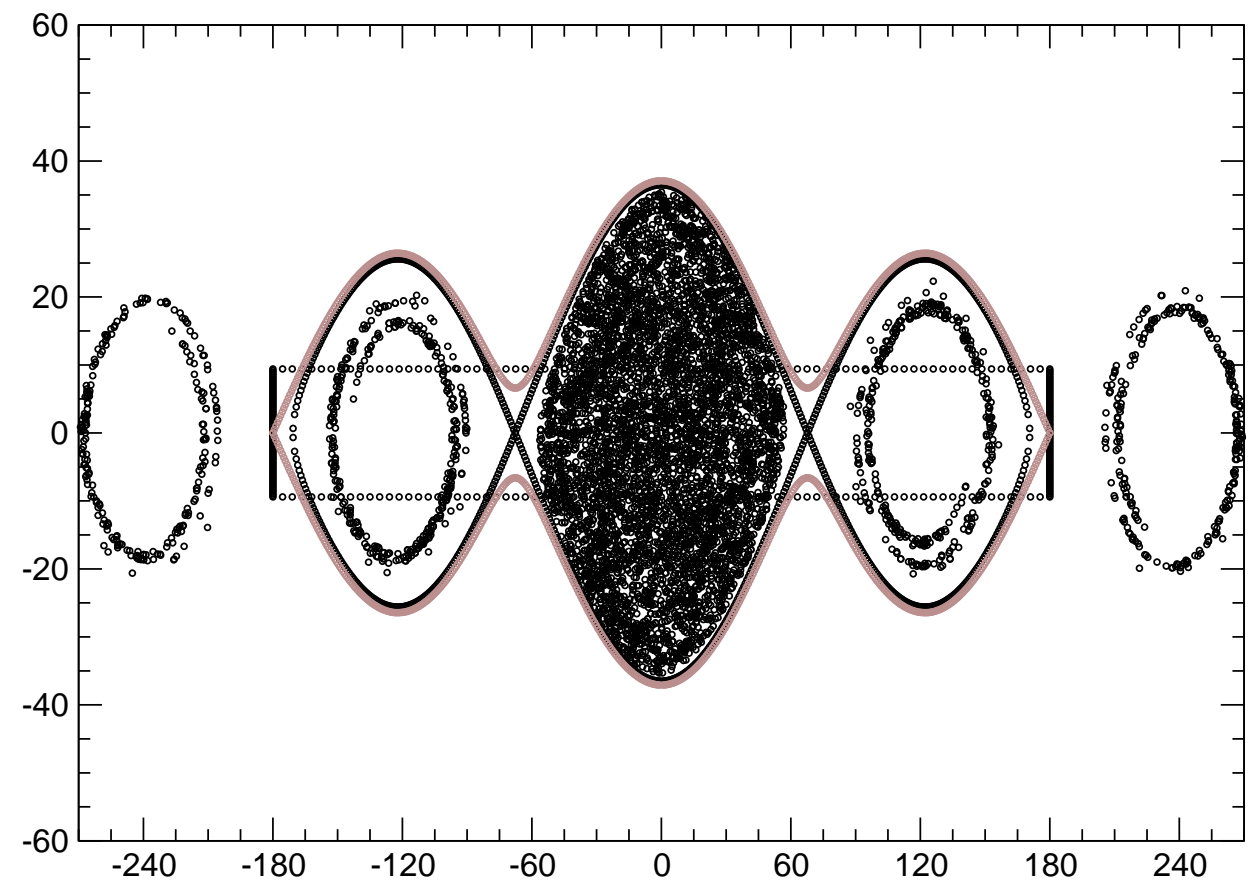

Figure 28: Here, with the harmonic 4 and 8 voltages held at $V_{L}=14 \mathrm{kV}$ and $V_{K}=22 \mathrm{kV}$ respectively, the harmonic 12 voltage has been raised from zero to $V_{A}=72 \mathrm{kV}$ in $4 \mathrm{~ms}$. The unstable fixed point phases are $\phi_{u}= \pm 67.7$ and $\phi_{u}= \pm 180$ degrees. 


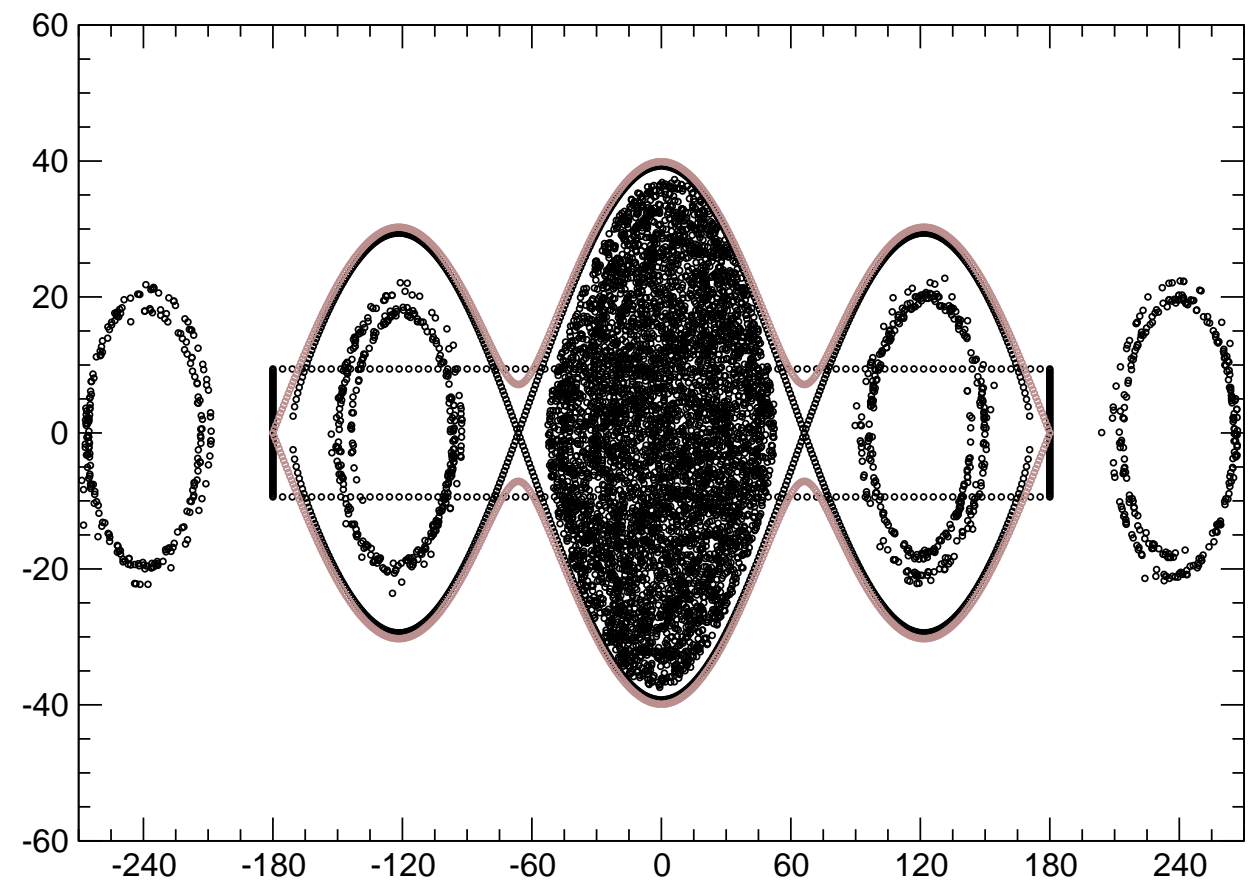

Figure 29: Here, with the harmonic 4 and 8 voltages held at $V_{L}=14 \mathrm{kV}$ and $V_{K}=22 \mathrm{kV}$ respectively, the harmonic 12 voltage has been raised from zero to $V_{A}=90 \mathrm{kV}$ in $5 \mathrm{~ms}$. The unstable fixed point phases are $\phi_{u}= \pm 66.3$ and $\phi_{u}= \pm 180$ degrees. 


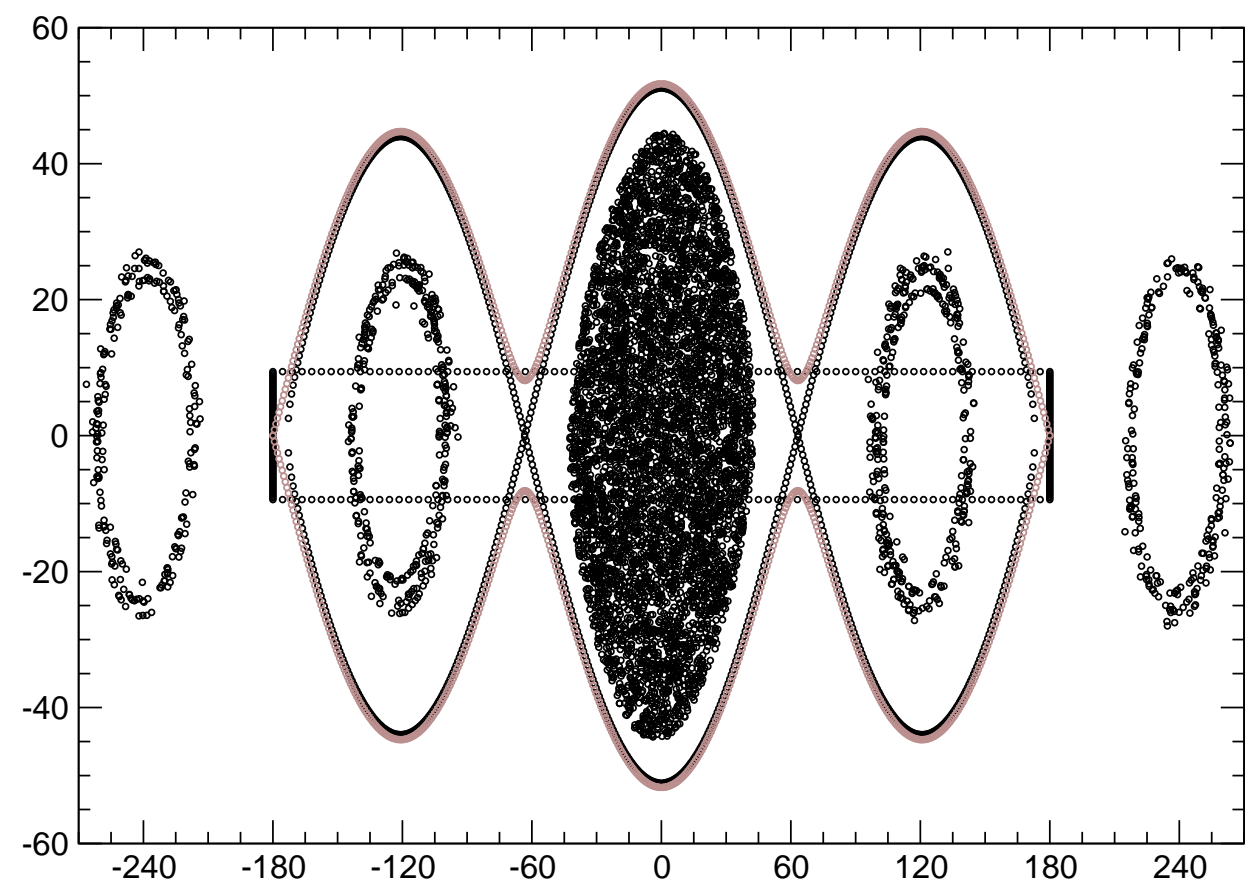

Figure 30: Here, with the harmonic 4 and 8 voltages held at $V_{L}=14 \mathrm{kV}$ and $V_{K}=22 \mathrm{kV}$ respectively, the harmonic 12 voltage has been raised from zero to $V_{A}=180 \mathrm{kV}$ in $10 \mathrm{~ms}$. The unstable fixed point phases are $\phi_{u}= \pm 63.2$ and $\phi_{u}= \pm 180$ degrees. The fractional momentum spread of the central bunch is $\Delta p / p= \pm 0.00462$. The fraction of Au77+ ions outside the central bucket is 0.180 . The fraction in the outermost buckets is 0.075 . 


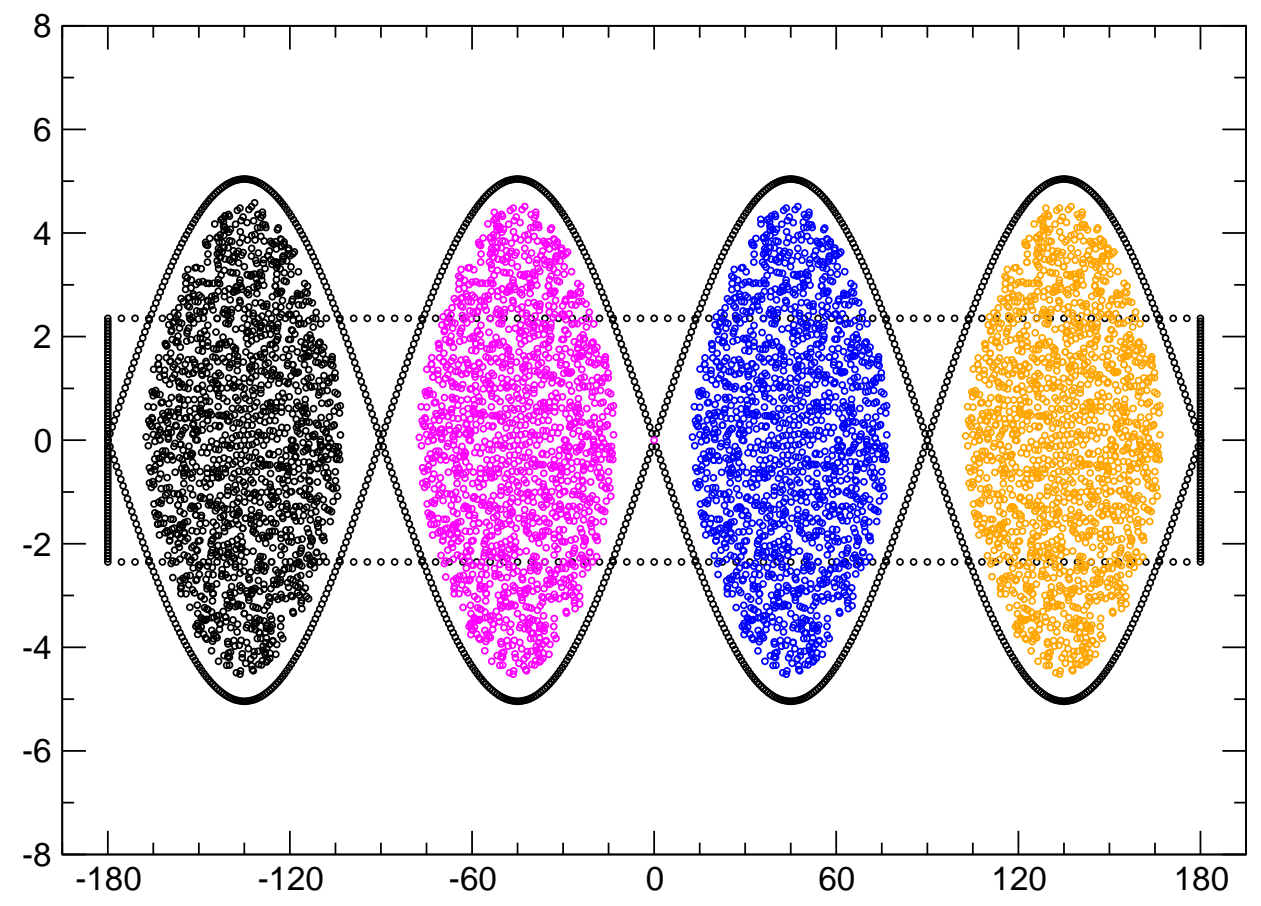

Figure 31: Four bunches of Au32+ ions in harmonic 4 buckets on the Booster merging porch. The harmonic 4 frequency is $4 f_{s}=1.860 \mathrm{MHz}$. The voltage is $V_{D}=2.0 \mathrm{kV}$. The black rectangle is the border of the uniform distribution used to make the bunches. The longitudinal emittance of the distribution is $0.15 \mathrm{eV}$ s per nucleon. One can see by inspection that the emittance of the 4 bunches is close to that of the initial distribution. The horizontal axis is the phase $\phi$ in degrees. The vertical axis is $W$ in units of eVs. 


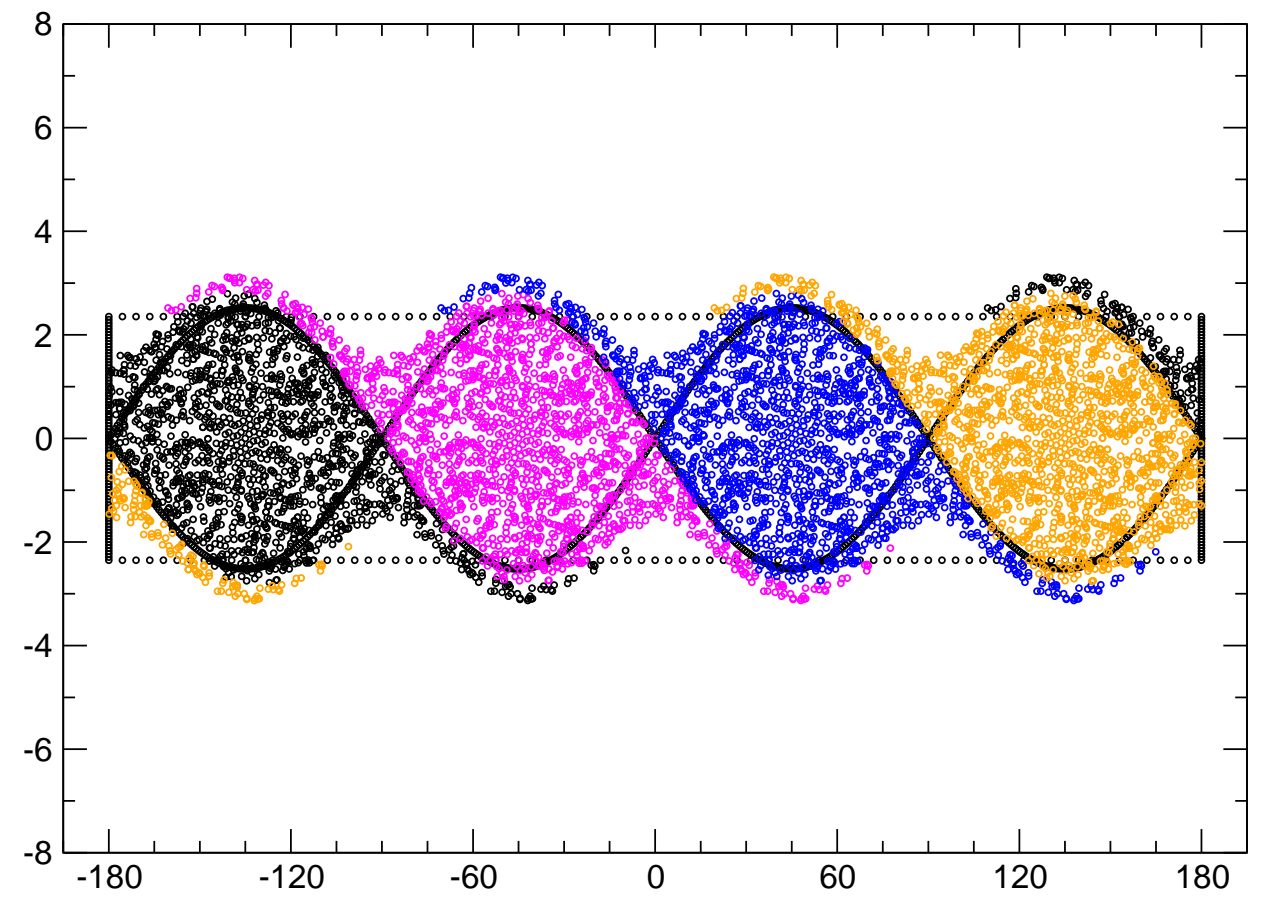

Figure 32: Halfway through the debunching of the bunches in Figure 31. 


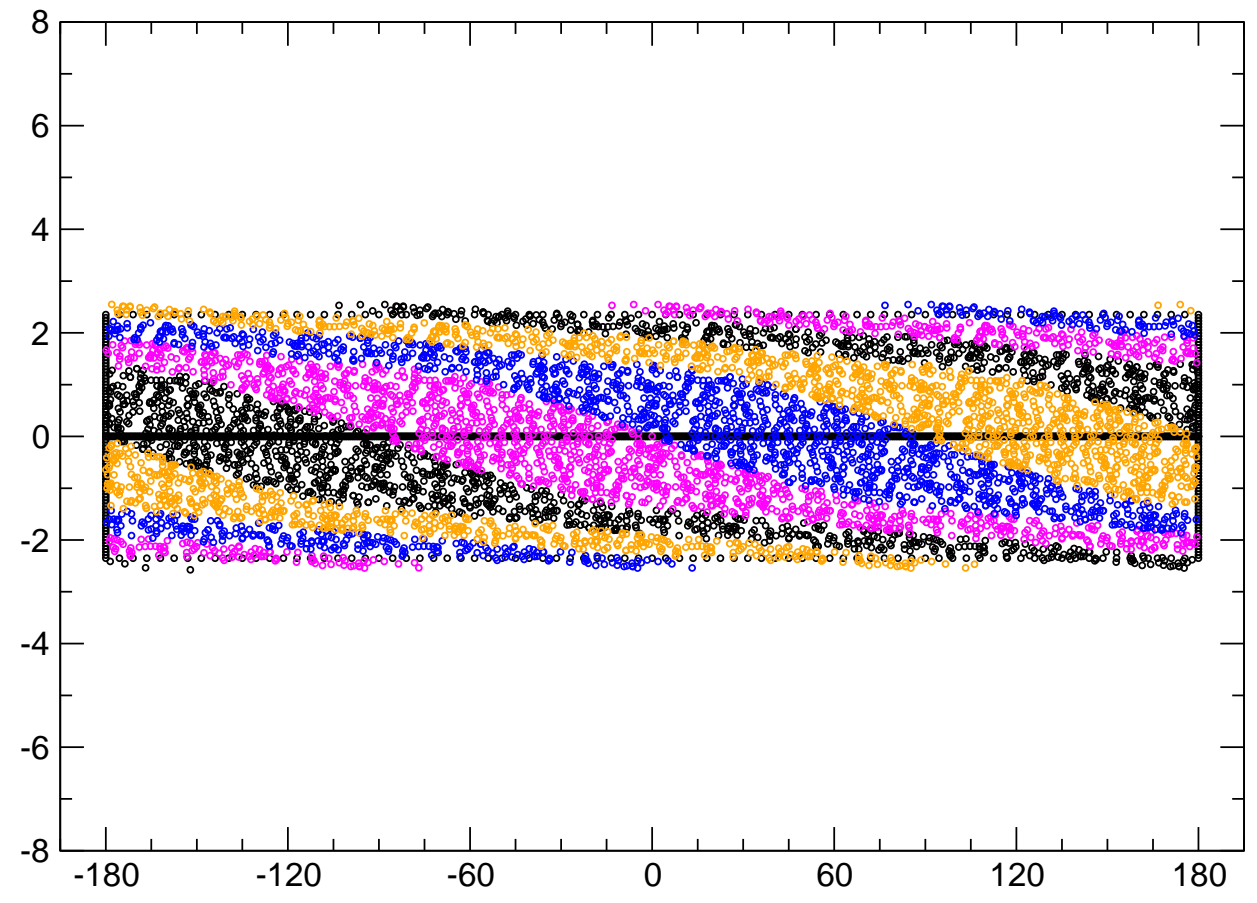

Figure 33: Completion of the debunching in $7.5 \mathrm{~ms}$. 


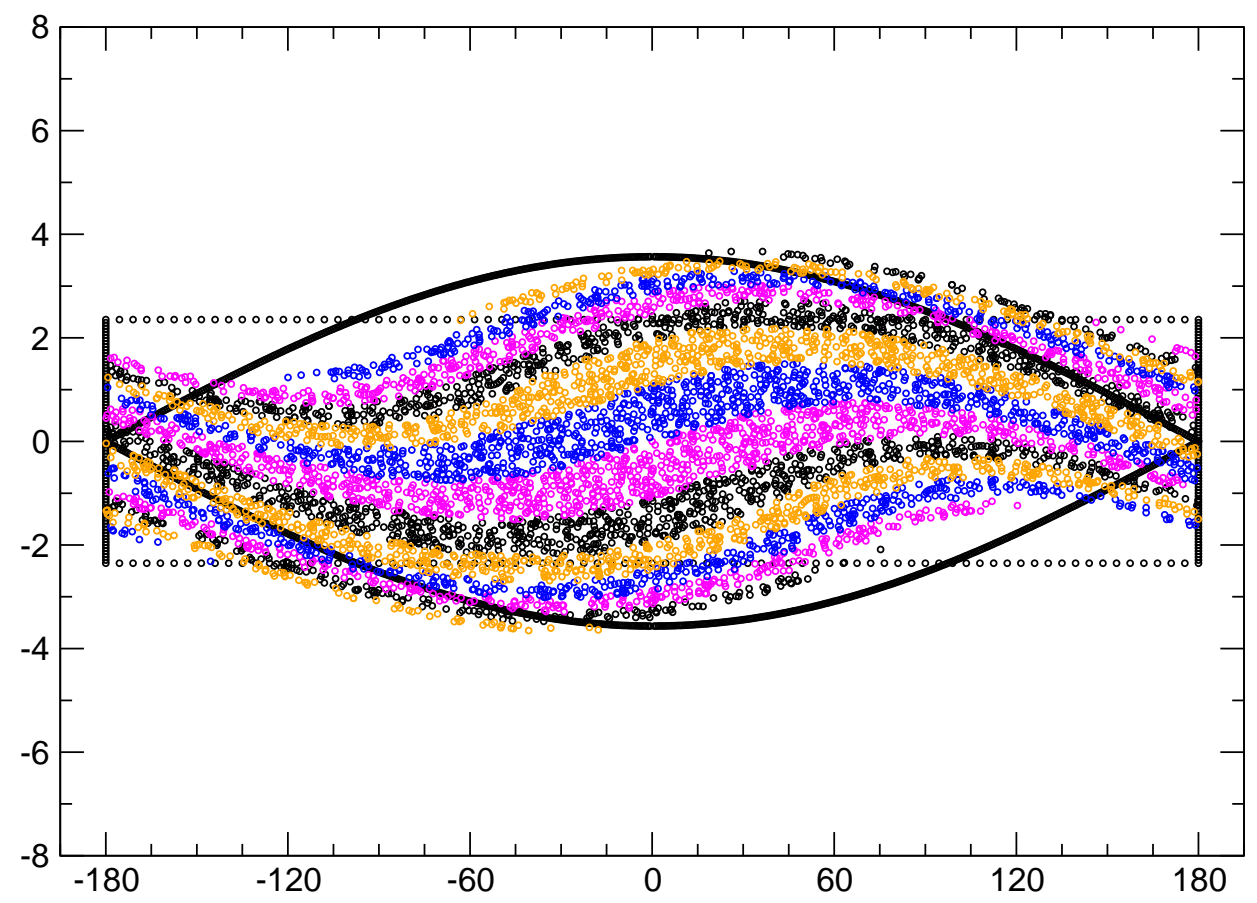

Figure 34: Halfway through the rebunching of the beam in Figure 33. 


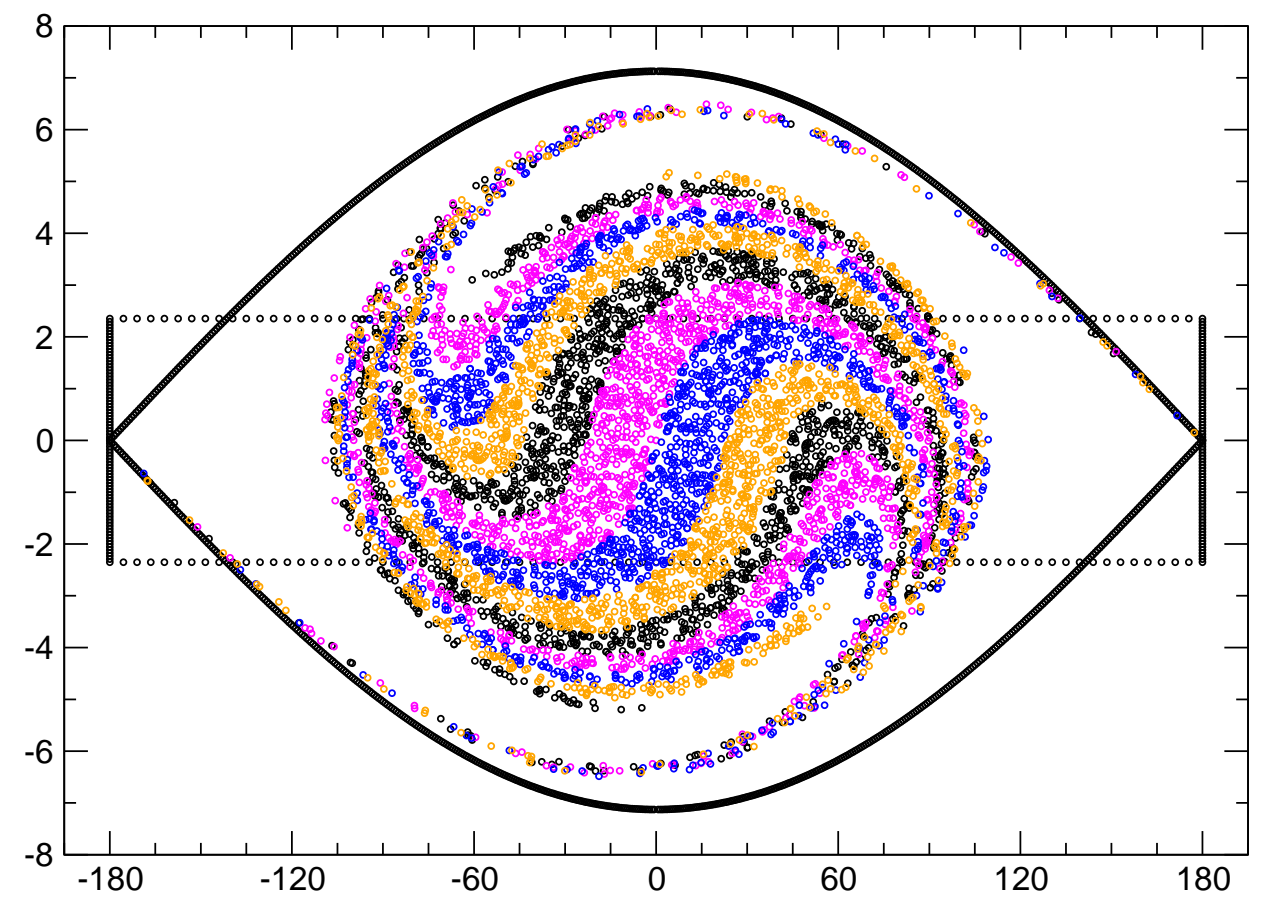

Figure 35: Completion of the rebunching in $7.5 \mathrm{~ms}$. 